\title{
Homotopy methods to compute equilibria in game theory
}

\author{
P. Jean-Jacques Herings • Ronald Peeters
}

Received: 17 October 2006 / Accepted: 16 January 2009 / Published online: 27 February 2009

(C) The Author(s) 2009. This article is published with open access at Springerlink.com

\begin{abstract}
This paper presents a survey of the use of homotopy methods in game theory. Homotopies allow for a robust computation of game-theoretic equilibria and their refinements. Homotopies are also suitable to compute equilibria that are selected by various selection theories. We present the relevant techniques underlying homotopy algorithms. We give detailed expositions of the Lemke-Howson algorithm and the van den Elzen-Talman algorithm to compute Nash equilibria in 2-person games, and the Herings-van den Elzen, Herings-Peeters, and McKelvey-Palfrey algorithms to compute Nash equilibria in general $n$-person games. We explain how the main ideas can be extended to compute equilibria in extensive form and dynamic games, and how homotopies can be used to compute all Nash equilibria.
\end{abstract}

Keywords Homotopy · Equilibrium computation · Non-cooperative games · Nash equilibrium

JEL Classification $\quad \mathrm{C} 62 \cdot \mathrm{C} 63 \cdot \mathrm{C} 72 \cdot \mathrm{C} 73$

\section{Introduction}

Economic research in the twentieth century has benefited greatly from the development of game theoretic tools. Typically, an economic situation is modeled as a game,

P. J. J. Herings and R. Peeters are financially supported by The Netherlands Organisation for Scientific Research.

P. J. J. Herings $(\varangle) \cdot$ R. Peeters

Department of Economics, Maastricht University, P.O. Box 616, 6200 MD Maastricht, The Netherlands

e-mail: p.herings@algec.unimaas.nl 
followed by an analysis of the outcomes of the game that satisfy a particular solution concept, typically that of Nash equilibrium or one of its refinements.

Since the analysis of Nash equilibria is key to the understanding of the situation at hand, much attention has been given to the development of methods by which Nash equilibria can be computed. We adhere to the view expressed in Judd (1997) that computational methods will serve an important role in the further development of economic theory, and in this case in the progress of game theory.

This survey provides an overview of a great variety of methods for Nash equilibrium computation that have been proposed. Although quite distinct in the specifics of the mathematical techniques used, all these methods share the property that they are guaranteed to converge to some Nash equilibrium. We argue that the homotopy idea is common to all these methods, and this idea should therefore be considered as the main driving force behind convergence. The homotopy idea is also extremely helpful in obtaining a thorough understanding of the methods themselves.

Advantages of the use of homotopy methods include their numerical stability, their potential to be globally rather than only locally convergent, their ability to potentially locate multiple solutions, and the insight they provide into properties of the solutions. Favorable by-products are that homotopies can be used for proving existence of an equilibrium and for the generic oddness of the number of equilibria. Similar to the view of Eaves and Schmedders (1999) “... inevitable and lasting role which homotopy methods will play in both theoretical and applied economic analysis" we believe in an important role for homotopy methods in game theory.

The homotopy idea is very simple. It consists of, first, the problem of interest, second an artificial problem that can easily be solved, and third a continuous transformation of the easy to solve artificial problem into the problem of interest. The homotopy idea then consists of solving the easy artificial problem first and then using the solution to solve the transformations of the easy problem until finally the problem of interest has been solved. Section 2 explains why a deep mathematical result known as Browder's fixed point theorem (Browder 1960), see also the extension by Mas-Colell (1974), is the fundamental reason that this approach works under very general assumptions.

We will refer to the easy to solve problem as the starting problem, the problem of interest as the terminal problem, and the problems created by the continuous transformation as the intermediate problems. The choice of different starting problems and the choice of different transformations of the starting problem to the terminal problem, creates different homotopy algorithms. We will explain all algorithms in these terms.

Our survey consists of two main parts. Part 1 (Sects. 5, 6) is devoted to the study of bimatrix games, whereas Part 2 (Sects. 8, 9, 10,11) treats general $n$-person games. The reason for this distinction is that in bimatrix games it is possible to find exact solutions for starting, intermediate and terminal problems, whereas general $n$-person games require some approximation method to find solutions.

Section 5 starts with a presentation of what is probably the best-known algorithm for the computation of Nash equilibria, the one of Lemke and Howson (1964). This algorithm finds a Nash equilibrium by solving a related linear complementarity problem. An alternative to the Lemke-Howson algorithm is provided by the algorithm of van den Elzen and Talman (1991), described in Sect. 6. The latter algorithm allows for an arbitrarily chosen starting point. 
The algorithms of Sects. 5 and 6 can be generalized to $n$-person games. For the Lemke-Howson procedure, such generalizations are given in Rosenmüller (1971) and Wilson (1971). Herings and van den Elzen (2002) extend the algorithm of van den Elzen and Talman (1991) to the $n$-person case. A difficulty to compute Nash equilibria in $n$-person games is posed by the non-linearity of the terminal problem. This makes it impossible to solve the intermediate problems exactly. Herings and van den Elzen (2002) tackle this problem by approximating the intermediate problems by piecewise linear ones, which can be solved exactly. Section 8 discusses the simplicial techniques that are needed for this approach. Section 9 presents the algorithm of Herings and Peeters (2001). This algorithm exploits the differentiability of game theoretic problems. The techniques needed for this approach come from differential topology and are presented in detail.

The Herings-van den Elzen and the Herings-Peeters algorithms, as well as the van den Elzen-Talman algorithm, compute the Nash equilibrium selected by the equilibrium selection theory of Harsanyi and Selten (1988). Another algorithm with a nice game theoretic interpretation is the one related to the quantal-response equilibrium of McKelvey and Palfrey (1995). This approach is explained in detail in Sect. 10. Although McKelvey and Palfrey do not give an algorithmic interpretation of their theory themselves, the results as presented in this survey make such an interpretation obvious.

Section 11 provides an overview of other homotopy methods that have been proposed to compute Nash equilibria. Section 12 discusses how the methods presented in the other sections can be extended to extensive form games and dynamic games. Section 13 explains how homotopies can be used to compute all Nash equilibria of a game. Section 14 concludes.

\section{Homotopy methods}

In topology, two continuous functions from one topological space to another are called homotopic if one can be "continuously deformed" into the other. Such a deformation is called a homotopy between the two functions. Formally, a homotopy between two continuous functions $f$ and $g$ from a topological space $X$ to a topological space $Y$ is defined to be a continuous function $H:[0,1] \times X \rightarrow Y$ such that, for all points $x$ in $X, H(0, x)=f(x)$ and $H(1, x)=g(x)$. If we think of the homotopy-parameter in $[0,1]$ as "time," then $H$ describes a "continuous deformation" of $f$ into $g$ : at time 0 we have the function $f$, at time 1 we have the function $g$.

A properly defined homotopy can be used to solve for the zeros of the function $g$ starting from the zeros of the function $f$ and gradually solve the nearby functions for increasing values of the homotopy parameter. This type of procedure is called a homotopy method. Just for the sake of convenience we consider the homotopy method from the perspective of finding a fixed point rather than a zero point in the remainder of this section. The following mathematical result due to Browder (1960) delivers the key insight for why homotopy methods converge under very general circumstances. 
Theorem 1 Let $S$ be a non-empty, compact, convex subset of $\mathbb{R}^{d}$ and let $H:[0,1] \times$ $S \rightarrow S$ be a continuous function. Then the set of fixed points, $F_{H}=\{(\lambda, s) \in$ $[0,1] \times S \mid s=H(\lambda, s)\}$ contains a connected set, $F_{H}^{\mathrm{c}}$, such that $(\{0\} \times S) \cap F_{H}^{\mathrm{c}} \neq \emptyset$ and $(\{1\} \times S) \cap F_{H}^{\mathrm{c}} \neq \emptyset$.

The set of fixed points $F_{H}$ of $H$ contains a connected subset $F_{H}^{\mathrm{c}}$ that intersects the two extremes: $\{0\} \times S$ and $\{1\} \times S$. This result suggests the following algorithm. Represent the problem for which a solution has to be computed, the terminal problem, as a fixed point problem, i.e. formulate a function $\left.H\right|_{\{1\} \times S}:\{1\} \times S \rightarrow S$ such that a fixed point of $H$ yields a solution to the terminal problem, where $\left.H\right|_{A}$ denotes the restriction of the function $H$ to the domain $A$. Formulate the starting problem as a fixed point problem, an artificial function $\left.H\right|_{\{0\} \times S}:\{0\} \times S \rightarrow S$ with usually a unique fixed point, say $s^{0}$, that can be computed easily. Define $\left.H\right|_{(0,1) \times S}:(0,1) \times S \rightarrow S$ in any way that makes $H$ continuous on $[0,1] \times S$. By Theorem 1 , the point $\left(0, s^{0}\right)$ is connected by $F_{H}^{\mathrm{c}}$ to a fixed point $\left(1, s^{1}\right)$ of $H$. Homotopy methods are methods to follow the connected set $F_{H}^{\mathrm{c}}$.

The following result, due to Mas-Colell (1974), shows that Theorem 1 can be generalized to the case of upper hemi-continuous correspondences.

Theorem 2 Let $S$ be a non-empty, compact, convex subset of $\mathbb{R}^{d}$ and let $H:[0,1] \times$ $S \rightarrow S$ be an upper hemi-continuous correspondence that is non-empty and convexvalued. Then the set of fixed points, $F_{H}=\{(\lambda, s) \in[0,1] \times S \mid s \in H(\lambda, s)\}$ contains a connected set, $F_{H}^{\mathrm{c}}$, such that $(\{0\} \times S) \cap F_{H}^{\mathrm{c}} \neq \emptyset$ and $(\{1\} \times S) \cap F_{H}^{\mathrm{c}} \neq \emptyset$.

Since the dimension of the domain of $H$ is one higher than the dimension of its range, it is usually possible to formulate appropriate regularity conditions for which the solution is a compact, piecewise differentiable one-dimensional manifold, i.e. a finite collection of arcs and loops. The algorithms for bimatrix games in Sects. 5 and 6 achieve this by a nondegeneracy condition. For $n$-person games, the appropriate regularity conditions are discussed in detail in Sect. 9. We will also present methods that work without such regularity conditions, most notably in Sect. 8 where we explain how lexicographic pivoting techniques can be used for the general case.

If the starting problem is constructed to have a unique fixed point, and appropriate regularity conditions are made, then there is a unique arc, also referred to as path or homotopy path, from this unique fixed point to a fixed point in $\{1\} \times S$. At this point it should be remarked that even if there is a unique arc, it may bend backwards, so under the interpretation of the homotopy parameter $t$ as time, following the homotopy path may involve going backward in time.

\section{Notation}

A finite $n$-person noncooperative game in normal form is a tuple $\Gamma=\left\langle N,\left\{S^{i}\right\}_{i \in N}\right.$, $\left.\left\{u^{i}\right\}_{i \in N}\right\rangle$, where $N=\{1, \ldots, n\}$ is the finite set of players, $S^{i}=\left\{s_{1}^{i}, \ldots, s_{m^{i}}^{i}\right\}$ is the finite set of strategies that player $i$ has at his disposal, and $u^{i}$ is a real valued function on the set of all possible strategy combinations $\chi_{i \in N} S^{i}$. A mixed strategy of player $i$ 
is a probability distribution on $S^{i}$. Thereto we define $\Sigma^{i}$ as the set of all probability distributions over $S^{i}$. For $\sigma^{i} \in \Sigma^{i}$, the probability assigned to pure strategy $s_{j}^{i}$ is given by $\sigma_{j}^{i}$. The payoff function is extended multi-linearly to the set of all mixed strategy combinations $\Sigma=X_{i \in N} \Sigma^{i}$. Given a mixed strategy combination $\sigma \in \Sigma$ and a mixed strategy $\bar{\sigma}^{i} \in \Sigma^{i}$, we denote by $\left(\sigma^{-i}, \bar{\sigma}^{i}\right)$ the mixed strategy combination that results from replacing $\sigma^{i}$ by $\bar{\sigma}^{i}$. A mixed strategy combination $\sigma \in \Sigma$ is said to be a Nash equilibrium of the game $\Gamma$ if $\sigma^{i}$ is a best response against $\sigma^{-i}$ for all $i \in N$. The set of Nash equilibria of the game $\Gamma$ is denoted by $\operatorname{NE}(\Gamma)$. We define the disjoint union of the pure strategies over all players by $S=\bigcup_{i \in N} S^{i}$ with $m=\sum_{i \in N} m^{i}$ as the cardinality of this set. All vectors in this survey are column vectors. The symbol $T$ is used for transposition.

The case $n=2$ is referred to as a bimatrix game. A bimatrix game can be represented by a pair of $m^{1} \times m^{2}$ matrices $\left(A^{1}, A^{2}\right)$, where $u^{1}\left(\sigma^{1}, \sigma^{2}\right)=\left(\sigma^{1}\right)^{\top} A^{1} \sigma^{2}$ and $u^{2}\left(\sigma^{1}, \sigma^{2}\right)=\left(\sigma^{1}\right)^{\top} A^{2} \sigma^{2}$.

The vector $e^{i}$ is the vector of length $m^{i}$ for which each element is equal to $1, e_{j}^{i}$ is the vector of length $m^{i}$ for which the $j$ th element is equal to 1 and all other elements 0 , and $0^{i}$ is the vector of length $m^{i}$ for which each element is equal to 0 . For a matrix $M$, we define by $M_{i} \bullet$ the $i$ th row and by $M_{\bullet j}$ the $j$ th column of the matrix $M$.

\section{Homotopies to compute Nash equilibria}

In this section we present the homotopies underlying the algorithms of Lemke-Howson (Sect. 5), van den Elzen-Talman (Sect. 6), Herings-van den Elzen and HeringsPeeters (Sects. 8, 9), and McKelvey-Palfrey (Sect. 10). The homotopies underlying the Herings-van den Elzen and the Herings-Peeters algorithm are identical. These algorithms only differ in the numerical technique that is used to follow the homotopy path. All the homotopies can be stated as correspondences from $[0,1] \times \Sigma$ into $\Sigma$. Since all algorithms are designed to find a Nash equilibrium of the game $\Gamma$, all homotopies share the same terminal problem. We define $\left.H\right|_{\{1\} \times \Sigma}$ as the product of the best response correspondences $\beta^{i}, i \in N$, in the game $\Gamma$, where $\beta^{i}(\sigma)=\arg \max _{\bar{\sigma}^{i} \in \Sigma^{i}} u^{i}\left(\sigma^{-i}, \bar{\sigma}^{i}\right)$. Clearly, the set of fixed points of the terminal problem coincides with the set of Nash equilibria of $\Gamma$.

The homotopies also have in common that, for each $t \in[0,1)$, the set of fixed points of $\left.H\right|_{\{t\} \times \Sigma}$ coincides with the set of Nash equilibria of a particular perturbation $\Gamma(t)$ of $\Gamma$. The correspondence $\left.H\right|_{\{t\} \times \Sigma}$ is defined as the product of best response correspondences in the game $\Gamma(t)$. The game $\Gamma(0)$ is chosen to be a game with a unique Nash equilibrium that is easily computed.

The homotopy $H$ of the previous paragraph is a correspondence. Using the techniques of Herings (2000) and Geanakoplos (2003), it is possible to define a continuous homotopy function with exactly the same homotopy path. This is achieved by replacing the best response correspondence of player $i$ by a function that assigns to $(t, \sigma)$ the unique strategy $\bar{\sigma}^{i}$ that maximizes the payoff in $\Gamma(t)$ to player $i$ minus a quadratic term given by the Euclidean distance to $\sigma^{i}$. The quadratic term is equal to zero at a fixed point, so fixed points do still correspond to Nash equilibria of $\Gamma(t)$. 


\subsection{The Lemke-Howson algorithm}

Let $\Gamma$ be a bimatrix game and let $\alpha$ be a bonus sufficiently large to make any pure strategy a dominant strategy once this bonus is given for the use of this strategy, for instance $\alpha=\max _{h, \sigma} u^{h}(\sigma)-\min _{h, \sigma} u^{h}(\sigma)+\varepsilon$, where $\varepsilon>0$. Given such an $\alpha$ and a pure strategy $s_{j}^{i} \in S^{i}$, we define, for $t \in[0,1]$, the bimatrix game $\Gamma(t)=$ $\left\langle N,\left\{S^{h}\right\}_{h \in N},\left\{v^{h}(t, \cdot)\right\}_{h \in N}\right\rangle$, where for player $i, v^{i}(t, s)$ equals $u^{i}(s)+(1-t) \alpha$ if $s^{i}=s_{j}^{i}$ and $u^{i}(s)$ otherwise; for the other player, denoted by $-i, v^{-i}(t, s)=u^{-i}(s)$.

The correspondence $H:[0,1] \times \Sigma \rightarrow \Sigma$ is defined by

$$
H(t, \sigma)=\prod_{h \in N} \beta^{h}(t, \sigma)
$$

where $\beta^{h}(t, \sigma)=\operatorname{argmax}_{\bar{\sigma}^{h} \in \Sigma^{h}} v^{h}\left(t, \sigma^{-h}, \bar{\sigma}^{h}\right)$ is the best response correspondence of player $h$ in game $\Gamma(t)$. The strategy profile consisting of strategy $s_{j}^{i}$ and a best response of the opponent is, by construction, an equilibrium of the game $\Gamma(0)$. By gradually increasing $t$ from 0 to 1 , the bonus on $s_{j}^{i}$ is gradually decreased from $\alpha$ to 0 , and the game of interest is reached. The set of strategy profiles of the homotopy path coincides precisely with the set of strategy profiles generated by the Lemke-Howson algorithm. Theorem 2 implies that a Nash equilibrium of $\Gamma(0)$ is connected to a Nash equilibrium of $\Gamma(1)=\Gamma$.

\subsection{The van den Elzen-Talman algorithm}

Let $\Gamma$ be a bimatrix game and let $v \in \Sigma$ be an arbitrarily chosen starting profile. For the van den Elzen-Talman algorithm one defines a family of subsets $\Sigma(t)$ of $\Sigma$, where $\Sigma(t)=(1-t)\{v\}+t \Sigma$ for $t \in[0,1]$. The set of strategy profiles $\Sigma(t)$ generates a game $\Gamma(t)$. By construction, $v$ is the unique Nash equilibrium of the game $\Gamma(0)$.

We define the correspondence $H:[0,1] \times \Sigma \rightarrow \Sigma$ by

$$
H(t, \sigma)=\prod_{i \in N} \beta_{\Sigma^{i}(t)}^{i}(\sigma),
$$

where $\beta_{\Sigma^{i}(t)}^{i}(\sigma)=\operatorname{argmax}_{\bar{\sigma}^{i} \in \Sigma^{i}(t)} u^{i}\left(\sigma^{-i}, \bar{\sigma}^{i}\right)$ is the best response correspondence of player $i$ in game $\Gamma$ with restricted set of mixed strategies $\Sigma^{i}(t)$. The set of strategy profiles of the resulting homotopy path coincides with the set of strategy profiles generated by the van den Elzen-Talman algorithm. Notice that the starting profile $v$ affects the homotopy for all values of $t$ below 1 . Theorem 2 implies that the unique Nash equilibrium of $\Gamma(0)$ is connected to a Nash equilibrium of $\Gamma(1)=\Gamma$.

\subsection{The Herings-van den Elzen algorithm and the Herings-Peeters algorithm}

Let $\Gamma$ be an $n$-person game and let $p \in \Sigma$ be a prior belief. For every $t \in[0,1]$, $\Gamma(t)$ is defined as the game $\left\langle N,\left\{S^{i}\right\}_{i \in N},\left\{v^{i}(t)\right\}_{i \in N}\right\rangle$, where the payoff function 
$v^{i}(t): \Sigma \rightarrow \mathbb{R}$ of player $i$ is defined by $v^{i}(t, \sigma)=t u^{i}(\sigma)+(1-t) u^{i}\left(p^{-i}, \sigma^{i}\right)$. The game $\Gamma(0)$ corresponds to a trivial game, where all players believe that their opponents play with probability 1 according to the prior belief. Any profile of best responses constitutes a Nash equilibrium of $\Gamma(0)$. The game $\Gamma(1)$ coincides with the original game $\Gamma$.

Next, we can define the homotopy correspondence $H:[0,1] \times \Sigma \rightarrow \Sigma$ by

$$
H(t, \sigma)=\prod_{i \in N} \beta^{i}(t, \sigma)
$$

where as before $\beta^{i}(t, \sigma)$ is the set of best responses of player $i$ in game $\Gamma(t)$ against strategy profile $\sigma^{-i}$. The set of strategy profiles of the resulting homotopy path coincides with the set of strategy profiles numerically followed by the Herings-van den Elzen and the Herings-Peeters algorithm. This homotopy coincides with the linear tracing procedure as defined by Harsanyi (1975). Notice that the prior belief $p$ affects the homotopy for all values of $t$ below 1. For the two-player case, choosing $p=v$, (3) and (2) are equivalent in the sense that, for $t>0,(t, \sigma)$ is a fixed point of (3) if and only if $(t,(1-t) p+t \sigma)$ is a fixed point of (2). Theorem 2 implies that the Nash equilibrium of $\Gamma(0)$ is connected to a Nash equilibrium of $\Gamma(1)=\Gamma$.

\subsection{The McKelvey-Palfrey algorithm}

Another homotopy method related to $n$-person games is derived from the quantal response theory of McKelvey and Palfrey (1995). McKelvey-Palfrey do not give an algorithmic interpretation of their theory themselves, but the results presented in this survey make such an interpretation obvious.

In the quantal response theory, the payoff to player $i$ when playing pure strategy $s_{j}^{i}$ against a mixed strategy combination $\sigma^{-i}$ is subject to error and is given by $v^{i}\left(\sigma^{-i}, s_{j}^{i}\right)=u^{i}\left(\sigma^{-i}, s_{j}^{i}\right)+\varepsilon_{j}^{i}(t)$, where $\varepsilon_{j}^{i}(t)$ represents the error term. Here player $i$ 's error vector $\varepsilon^{i}(t)$ is distributed according to a distribution with density function $\varphi^{i}$ such that $\mathbb{E}\left(\varepsilon_{j}^{i}(t)\right)=0$. We refer the reader to Sect. 10 for more details on quantal response theory.

A particular example concerns the case where $\varphi^{i}$ corresponds to the extreme value distribution. In this case, player $i$ has the statistical response function defined by

$$
\beta_{j}^{i}(\lambda, \sigma)=\frac{\exp \left(\lambda u^{i}\left(\sigma^{-i}, s_{j}^{i}\right)\right)}{\sum_{k=1}^{m^{i}} \exp \left(\lambda u^{i}\left(\sigma^{-i}, s_{k}^{i}\right)\right)} \quad\left(s_{j}^{i} \in S^{i}, i \in N\right) .
$$

where $\beta_{j}^{i}$ should be interpreted as the weight that player $i$ puts on pure strategy $s_{j}^{i}$. Here $\lambda$ is a parameter of the extreme value distribution. Values of $\lambda$ equal to zero mean that strategies consists of all error, and errors disappear when $\lambda$ tends to infinity.

Next, we can define the homotopy correspondence $H:[0,1] \times \Sigma \rightarrow \Sigma$ by

$$
H(t, \sigma)=\prod_{i \in N}\left\{\beta^{i}(t, \sigma)\right\}, \quad(t, \sigma) \in[0,1) \times \Sigma,
$$


Table 1 Algorithms described in this survey

\begin{tabular}{lllll}
\hline Section & Algorithm & Homotopy & \# Players & Technique \\
\hline 5 & Lemke-Howson & $(1)$ & 2 & Complementary pivoting \\
6 & van den Elzen-Talman & $(2)$ & 2 & Complementary pivoting \\
8 & Herings-van den Elzen & $(3)$ & $n$ & Simplicial \\
9 & Herings-Peeters & $(3)$ & $n$ & Predictor-corrector \\
10 & McKelvey-Palfrey & $(4)$ & $n$ & Predictor-corrector \\
\hline
\end{tabular}

where $\beta^{i}(t, \sigma)$ is the best response function for an extreme value distribution with parameter $t /(1-t)$, and

$$
H(1, \sigma)=\prod_{i \in N} \beta^{i}(\sigma)
$$

the product of best response correspondences in the game of interest $\Gamma$.

When $t=0$, there is a unique fixed point. At the fixed point, each player chooses each of his pure strategies with equal probability. Theorem 2 implies that this fixed point is connected to a Nash equilibrium of $\Gamma$.

\subsection{Homotopy continuation}

For bimatrix games, the homotopy path is piecewise linear. It is possible to generate the homotopy path by a finite sequence of complementary pivoting steps. For general $n$-person games, the homotopy path has to be traced numerically. There are two fundamental methods of doing so: simplicial methods and predictor-corrector methods. Simplicial methods exactly follow approximate solution curves, whereas predictorcorrector methods approximately follow exact solution curves. For a detailed overview on path-tracking methods the reader is referred to Garcia and Zangwill (1981) and Allgower and Georg (1990).

The different algorithms mentioned throughout this section use different ways to trace the path that is determined by the respective homotopy. Table 1 shows for each of these algorithms the section in which it is explained in detail, the homotopy that is followed, and the path-following method that is used.

\section{Bimatrix games: the Lemke-Howson algorithm}

Recall that the disjoint union of the pure strategies over all players is denoted by $S$, and the cardinality of this set by $m$. Any mixed strategy $\sigma^{i}$ is assigned a set of labels in $S$. In particular, $\sigma^{i}$ is labeled by the pure strategies that are played with probability zero and the pure strategies of the opponent that are best responses to it:

$$
\begin{aligned}
& L^{1}\left(\sigma^{1}\right)=\left\{s_{j}^{1} \in S^{1} \mid \sigma_{j}^{1}=0\right\} \cup\left\{s_{j}^{2} \in S^{2} \mid\left(A_{\bullet j}^{2}\right)^{\top} \sigma^{1} \geq\left(A_{\bullet k}^{2}\right)^{\top} \sigma^{1}\left(s_{k}^{2} \in S^{2}\right)\right\}, \\
& L^{2}\left(\sigma^{2}\right)=\left\{s_{j}^{2} \in S^{2} \mid \sigma_{j}^{2}=0\right\} \cup\left\{s_{j}^{1} \in S^{1} \mid A_{\bullet j}^{1} \sigma^{2} \geq A_{\bullet k}^{1} \sigma^{2}\left(s_{k}^{1} \in S^{1}\right)\right\} .
\end{aligned}
$$


A profile of mixed strategies $\sigma=\left(\sigma^{1}, \sigma^{2}\right)$ is labeled by the union of the labels of $\sigma^{1}$ and $\sigma^{2}$ :

$$
L(\sigma)=L^{1}\left(\sigma^{1}\right) \cup L^{2}\left(\sigma^{2}\right) .
$$

A profile of mixed strategies $\sigma$ is called completely labeled if $L(\sigma)=S$ and called $s_{j}^{i}$-almost completely labeled if $L(\sigma) \cup\left\{s_{j}^{i}\right\}=S$. The following result can easily be shown.

Theorem 3 A mixed strategy pair $\left(\sigma^{1}, \sigma^{2}\right) \in \Sigma$ constitutes a Nash equilibrium of the bimatrix game $\left(A^{1}, A^{2}\right)$ if and only if it is completely labeled.

A bimatrix game with the property that any $\sigma^{i} \in \Sigma^{i}$ has at most $m^{i}$ labels is called nondegenerate. For the moment, we will restrict the analysis to the class of nondegenerate games. The following result is stated in von Stengel (2002) as Theorem 2.7.

Theorem 4 In a nondegenerate bimatrix game $\left(A^{1}, A^{2}\right)$ only finitely many points in $\Sigma^{1}$ have $m^{1}$ labels and only finitely many points in $\Sigma^{2}$ have $m^{2}$ labels.

It follows as a consequence of this theorem that in a nondegenerate bimatrix game there are finitely many points in $\Sigma^{1} \times \Sigma^{2}$ that have $m^{1}+m^{2}$ labels. Consequently, there are finitely many points that are completely labeled and hence finitely many Nash equilibria.

Consider the homotopy as defined in (1). The strategy profile $\sigma$ belongs to $H(t, \sigma)$ if and only if $\sigma$ is a Nash equilibrium of $\Gamma(t)$. We argue next that if $\sigma$ is a fixed point, then $L(\sigma) \cup\left\{s_{j}^{i}\right\}=S$, so $\sigma$ is $s_{j}^{i}$-almost completely labeled. Indeed, a pure strategy unequal $s_{j}^{i}$ is either played with probability 0 or is a best response in $\Gamma(t)$, so certainly a best response in $\Gamma(1)$. Conversely, if $\sigma$ is $s_{j}^{i}$-almost completely labeled, then $\sigma \in H(t, \sigma)$ for some $t \in[0,1]$. Also, $\sigma$ is completely labeled if and only if $\sigma \in H(1, \sigma)$.

The algorithm of Lemke and Howson (1964) boils down to following the homotopy-path, i.e. following a path of $s_{j}^{i}$-almost completely labeled strategy profiles. For nondegenerate games, the set of points $(t, \sigma)$ for which $\sigma \in H(t, \sigma)$ consists of a finite number of piecewise linear arcs and loops. Each arc has two endpoints, either both being Nash equilibria of $\Gamma$, or one being a Nash equilibrium of $\Gamma$ and the other of $\Gamma(0)$. The latter arc corresponds to the homotopy-path. Loops do not contain any Nash equilibria. It follows immediately that the number of Nash equilibria of a nondegenerate game is odd.

In a nondegenerate game, player $-i$ has a unique best response to $s_{j}^{i}$, say $s_{k}^{-i}$. The pure strategy profile $\left(s_{j}^{i}, s_{k}^{-i}\right)$ is the unique Nash equilibrium of $\Gamma(0)$, and is $s_{j}^{i}$ almost completely labeled. Clearly, $\left(s_{j}^{i}, s_{k}^{-i}\right) \in H\left(0,\left(s_{j}^{i}, s_{k}^{-i}\right)\right)$, and for sufficiently small values of $t,\left(s_{j}^{i}, s_{k}^{-i}\right) \in H\left(t,\left(s_{j}^{i}, s_{k}^{-i}\right)\right)$. Either $\left(s_{j}^{i}, s_{k}^{-i}\right)$ is a Nash equilibrium of $\Gamma$, and the homotopy-path equals the straight line-segment between $\left(0,\left(s_{j}^{i}, s_{k}^{-i}\right)\right)$ and $\left(1,\left(s_{j}^{i}, s_{k}^{-i}\right)\right)$, or $\left(s_{j}^{i}, s_{k}^{-i}\right)$ is not a Nash equilibrium of $\Gamma$, and for some value of $t<1$, say $t^{\prime}$, a second pure strategy of player $i$, say $s_{j^{\prime}}^{i}$, is a best response against $s_{k}^{-i}$ 
in game $\Gamma\left(t^{\prime}\right)$. Following the homotopy-path now corresponds to generating a new line-segment of points $(t, \sigma)$ with the property that $\sigma^{i}$ puts positive probability only on $s_{j}^{i}$ and $s_{j^{\prime}}^{i}$, both pure strategies are best responses in $\Gamma(t)$ to $s_{k}^{-i}$, and $s_{k}^{-i}$ is a best response in $\Gamma(t)$ to $\sigma^{i}$. After a finite number of such steps, the algorithm terminates with a Nash equilibrium of $\Gamma$ as soon as the value $t=1$ is reached.

The algebraic technique that generates the path of $s_{j}^{i}$-almost complete strategy profiles is known as complementary pivoting. As in linear programming, pivoting refers to the change of a basis in a linear system of equations that describes a polyhedron. Contrary to linear programming, the determination of the variable that enters the basis is not determined by the improvement in the objective function, but by complementarity considerations. For a self-contained description of complementary pivoting, we refer the reader to von Stengel (2002). We will illustrate the technique of complementary pivoting in more detail in our exposition of the van den Elzen-Talman algorithm.

We continue our explanation of the Lemke-Howson algorithm by following its exposition in Shapley (1974). This exposition consists of a compact and elegant description of the endpoints of each linear segment in the piecewise linear homotopy-path.

Consider a nondegenerate bimatrix game $\left(A^{1}, A^{2}\right)$ and let $G^{i}$ (for $i=1,2$ ) be the undirected graph whose vertices are the points from $\Sigma^{i}$ with $m^{i}$ labels, and an additional vertex $0^{i}$ having all strategies of player $i$ as label $\left(L^{i}\left(0^{i}\right)=S^{i}\right) .{ }^{1}$ Any two vertices of $G^{i}$ are joined by an edge if they have exactly $m^{i}-1$ labels in common (and thus differ in one label precisely).

Let $G=G^{1} \times G^{2}$ be the product graph with the vertices being all pairs of vertices of $G^{1}$ and $G^{2}$ and the edges being all vertex-edge pairs with vertices coming from $G^{1}$ and edges from $G^{2}$ or vice versa. In line with the definitions above, an edge of $G$ is called $s_{j}^{i}$-almost completely labeled if the two vertices that are connected by it are $s_{j}^{i}$-almost completely labeled.

A first observation is that for every $s_{j}^{i}$ any completely labeled vertex $\left(\sigma^{1}, \sigma^{2}\right)$ in $G$, that is any Nash equilibrium and the additional vertex $\left(0^{1}, 0^{2}\right)$, is adjacent to exactly one $s_{j}^{i}$-almost completely labeled vertex $\left(\tilde{\sigma}^{1}, \tilde{\sigma}^{2}\right)$. If $s_{j}^{i}$ is a label of $\sigma^{1}$, then $\sigma^{1}$ is adjacent to $\tilde{\sigma}^{1}$ in $G^{1}$ (sharing the same remaining $m^{1}-1$ labels) and $\sigma^{2}=\tilde{\sigma}^{2}$. If $s_{j}^{i}$ is a label of $\sigma^{2}$, then $\sigma^{2}$ is adjacent to $\tilde{\sigma}^{2}$ in $G^{2}$ (sharing the same remaining $m^{2}-1$ labels) and $\sigma^{1}=\tilde{\sigma}^{1}$. A second observation is that any vertex $\left(\sigma^{1}, \sigma^{2}\right)$ in $G$ that is $s_{j}^{i}$-almost completely labeled but not completely labeled, is adjacent to exactly two $s_{j}^{i}$-almost completely labeled vertices in $G$. A vertex $\left(\sigma^{1}, \sigma^{2}\right)$ in $G$ can only be $s_{j}^{i}$-almost completely labeled if $\sigma^{1}$ and $\sigma^{2}$ both have one other label $s_{k}^{h}$ in common. One neighbor of $\left(\sigma^{1}, \sigma^{2}\right)$ is $\left(\tilde{\sigma}^{1}, \sigma^{2}\right)$ with $\tilde{\sigma}^{1}$ being adjacent to $\sigma^{1}$ by an $s_{k}^{h}$-almost completely labeled edge in $G^{1}$. The other is $\left(\sigma^{1}, \tilde{\sigma}^{2}\right)$ with $\tilde{\sigma}^{2}$ being adjacent to $\sigma^{2}$

\footnotetext{
1 Our explanation of the Lemke-Howson algorithm based on homotopies does not need the additional vertex $0^{i}$. The strategy profile $\left(s_{j}^{i}, s_{k}^{-i}\right)$ in our exposition can be used to replace the additional vertex.
} 
Fig. 1 Example

\begin{tabular}{l|cc} 
& \multicolumn{1}{c}{$s_{1}^{2}$} & $s_{2}^{2}$ \\
\cline { 2 - 3 }$s_{1}^{1}$ & 2,2 & 1,4 \\
$s_{2}^{1}$ & 1,4 & 4,0 \\
\cline { 2 - 3 } &
\end{tabular}

by an $s_{k}^{h}$-almost completely labeled edge in $G^{2}$. For the following result, see Lemke and Howson (1964) or Shapley (1974).

Theorem 5 Let $\left(A^{1}, A^{2}\right)$ be a nondegenerate bimatrix game and $s_{j}^{i}$ be a label in $S$. Then the set of $s_{j}^{i}$-almost completely labeled vertices and edges in $G$ consists of disjoint arcs and loops. The end-points of the arcs are the completely labeled vertices (the equilibria of the game) and the completely labeled vertex $\left(0^{1}, 0^{2}\right)$ (the artificial equilibrium). The number of Nash equilibria of the game is odd.

The algorithm of Lemke-Howson starts in the artificial equilibrium $\left(0^{1}, 0^{2}\right)$ that has all labels. For given label $s_{j}^{i}$ a path consisting of $s_{j}^{i}$-almost completely labeled edges and vertices is followed that terminates at a Nash equilibrium of the game. From a computational point of view, moving from one almost completely labeled vertex pair to another, is equivalent to making a linear programming pivot step. We will explain this in more detail for the van den Elzen-Talman algorithm presented in Sect. 6.

Essentially, the Lemke-Howson algorithm terminates at an equilibrium because for a given label $s$, the set of almost $s$-almost completely labeled edges is a graph where every vertex has only one or two neighbors, so this is clearly a collection of paths and cycles. The endpoints of the paths come in pairs and are the equilibria, including the artificial equilibrium, so the number of non-artificial equilibria is odd. A vivid image is the "haunted house", which is a house with only one entry from the outside and where every room has only one or two doors. By entering from the outside and subsequently leaving each entered room via the second door of that room, one will eventually reach a room with only one door only or continues ad infinitum. The latter cannot happen, since it would imply that the first room visited for the second time has three doors. The outside entrance corresponds to the artificial equilibrium, and the rooms with one door to Nash equilibria of interest.

Consider the game of Fig. 1. This game possesses a unique Nash equilibrium: the mixed strategy profile $\left(\bar{\sigma}^{1}, \bar{\sigma}^{2}\right)=\left(\left(\frac{2}{3}, \frac{1}{3}\right),\left(\frac{3}{4}, \frac{1}{4}\right)\right)$.

Figure 2 contains the graphs $G^{1}$ and $G^{2}$ for this game. The vertices are indicated by the (mixed) strategies, the labels by the pure strategies with quotation marks. The path of $s_{1}^{1}$-almost completely labeled vertices and edges connects the artificial equilibrium $\left(0^{1}, 0^{2}\right)$ via the vertices $\left(s_{1}^{1}, 0^{2}\right),\left(s_{1}^{1}, s_{2}^{2}\right),\left(\bar{\sigma}^{1}, s_{2}^{2}\right)$ to the equilibrium $\left(\bar{\sigma}^{1}, \bar{\sigma}^{2}\right)$. Our exposition of Lemke-Howson using the homotopy approach would skip the two artificial vertices $\left(0^{1}, 0^{2}\right)$ and $\left(s_{1}^{1}, 0^{2}\right)$, and starts directly with $\left(s_{1}^{1}, s_{2}^{2}\right)$.

In game theory degeneracy is not always a non-generic phenomenon. For instance, for a normal form representation of a game in extensive form, degeneracy is the rule rather than the exception, even if the payoffs in the extensive form game are randomly chosen. But also in other normal form games, representing certain economic situations, degeneracy can easily occur, simply because payoffs are not randomly chosen but reflect some structure that is present in the economic model. Lemke and Howson (1964) resolve degeneracy by perturbing the game and show that an equilibrium for the 
Fig. 2 Graphical illustration of the Lemke-Howson algorithm corresponding to the game in

Fig. 1. The $s_{1}^{1}$-almost completely labeled path is given by steps I-IV
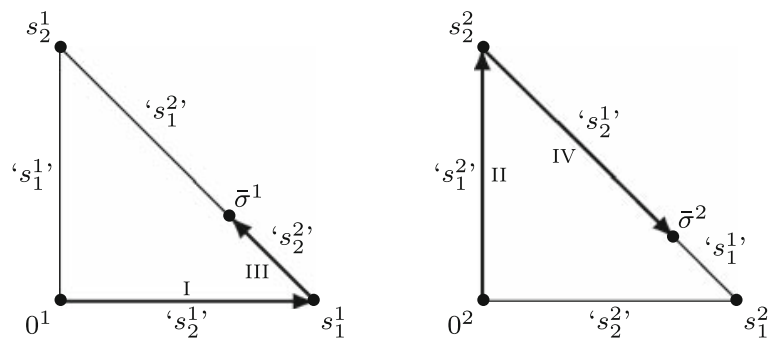

perturbed game defines an equilibrium for the original game. Wilson (1992) amends the Lemke-Howson algorithm to handle degenerate games by using lexicographic pivoting techniques. The resulting algorithm can be used to compute a set of simply stable equilibria. ${ }^{2}$ We will illustrate the use of lexicographic pivoting techniques in Sect. 8 when dealing with the Herings-van den Elzen algorithm.

The Lemke-Howson algorithm can be extended to the class of polymatrix games. These are games where for each player the payoffs are additive over the bilateral interactions with the other players. Complementary pivoting has been applied to polymatrix games by Yanovskaya (1986), Howson (1972), Eaves (1973), and Howson and Rosenthal (1974).

\section{Bimatrix games: the van den Elzen-Talman algorithm}

The Lemke-Howson algorithm always starts in a pure strategy profile, where for one player a pure strategy is randomly chosen, and the other player uses a best response. van den Elzen and Talman (1991) developed an algorithm similar to the LemkeHowson algorithm that allows for an arbitrary mixed strategy profile as the starting point. In von Stengel et al. (2002) it has been shown that the van den Elzen-Talman algorithm is equivalent to Lemke's algorithm (Lemke 1965) for a specific auxiliary vector.

The advantage of an arbitrary starting point is twofold. First, often some reasonable guess of the pure strategies that should be played in an equilibrium is available. Then it is natural to take as a starting point of the algorithm a strategy that puts only weight on such strategies. Secondly, if there is an interest in detecting whether a given game has multiple Nash equilibria, or there is a desire to compute several Nash equilibria if there exist multiple ones, the flexibility of the starting point is a desirable feature. We would like to stress that the analysis that follows can be adapted in a straightforward way to the Lemke-Howson algorithm.

A subset $B$ of $S$ is called admissible if $B \cap S^{i} \neq \emptyset$ for all $i \in N$, so an admissible subset $B$ of $S$ contains at least one pure strategy for each player. We would like to warn the reader that our use of admissible subset is unrelated to the concept of admissible strategy, which in game theory means a strategy that is not weakly dominated.

\footnotetext{
2 A set of equilibria is called stable if every game nearby has equilibria nearby. Simple stability results if the perturbations are not arbitrary but are restricted to certain systematic ways that are easily captured computationally.
} 
Let a starting point $v \in \Sigma$ be given. Then $\sigma \in H(t, \sigma)$ for the homotopy defined in (2) if and only if there is an admissible subset $B$ of $S$ and vectors $\tau^{i}, \lambda^{i} \in \mathbb{R}_{+}^{m^{i}}$, and $\mu^{i} \in \mathbb{R}$ such that

$$
\begin{array}{ll}
\sigma_{j}^{i}=(1-t) v_{j}^{i}+\tau_{j}^{i} & \left(s_{j}^{i} \in S\right) \\
u^{i}\left(\sigma^{-i}, s_{j}^{i}\right)+\lambda_{j}^{i}=\mu^{i} & \left(s_{j}^{i} \in S\right) \\
\sum_{s_{j}^{i} \in S^{i}} \tau_{j}^{i}=t & (i \in N) \\
\tau_{j}^{i}=0 & \left(s_{j}^{i} \notin B\right) \\
\lambda_{j}^{i}=0 & \left(s_{j}^{i} \in B\right) .
\end{array}
$$

Suppose $(t, \sigma, \tau, \lambda, \mu)$ is a solution to (5) (notice that not all $B$ admit solutions in general). The variable $\mu^{i}$ represents the maximum payoff that player $i$ can get against strategy $\sigma^{-i}$. The pure strategies in $B$ are all best responses. The $\lambda_{j}^{i}$, s corresponding to best responses are therefore equal to zero. For strategies not in $B$, the corresponding variable $\tau_{j}^{i}$ is equal to zero.

For bimatrix games the system above is linear and the second line of it can be replaced by

$$
\begin{aligned}
A_{j}^{1} \bullet \sigma^{2}+\lambda_{j}^{1} & =\mu^{1} & & \left(s_{j}^{1} \in S^{1}\right) \\
\left(\sigma^{1}\right)^{\top} A_{\bullet j}^{2}+\lambda_{j}^{2} & =\mu^{2} & & \left(s_{j}^{2} \in S^{2}\right) .
\end{aligned}
$$

By substitution of the first line of (5) into these new equations we end up with the following system of $2\left(m^{1}+m^{2}+1\right)$ linear equations and $2\left(m^{1}+m^{2}+1\right)+1$ variables:

$$
\begin{array}{ll}
A_{j \bullet}^{1}\left((1-t) v^{2}+\sum_{s_{k}^{2} \in B} \tau_{k}^{2} e_{k}^{2}\right)+\lambda_{j}^{1}=\mu^{1} & \left(s_{j}^{1} \in S^{1}\right) \\
\left((1-t) v^{1}+\sum_{s_{k}^{1} \in B} \tau_{k}^{1} e_{k}^{1}\right)^{\top} A_{\bullet j}^{2}+\lambda_{j}^{2}=\mu^{2} & \left(s_{j}^{2} \in S^{2}\right) \\
\sum_{s_{j}^{i} \in S^{i}} \tau_{j}^{i}=t & (i \in N) \\
\tau_{j}^{i}=0 & \left(s_{j}^{i} \notin B\right) \\
\lambda_{j}^{i}=0 & \left(s_{j}^{i} \in B\right) .
\end{array}
$$

Or, in matrix-vector notation,

$$
\begin{aligned}
& (1-t)\left(\begin{array}{c}
A^{1} v^{2} \\
\left(A^{2}\right)^{\top} v^{1} \\
1 \\
1
\end{array}\right)+\sum_{s_{k}^{1} \in B} \tau_{k}^{1}\left(\begin{array}{c}
0^{1} \\
\left(A_{k \bullet}^{2}\right)^{\top} \\
1 \\
0
\end{array}\right)+\sum_{s_{k}^{2} \in B} \tau_{k}^{2}\left(\begin{array}{c}
A_{\bullet^{1}}^{1} \\
0^{2} \\
0 \\
1
\end{array}\right) \\
& +\sum_{s_{k}^{1} \notin B} \lambda_{k}^{1}\left(\begin{array}{c}
e_{k}^{1} \\
0^{2} \\
0 \\
0
\end{array}\right)+\sum_{s_{k}^{2} \notin B} \lambda_{k}^{2}\left(\begin{array}{c}
0^{1} \\
e_{k}^{2} \\
0 \\
0
\end{array}\right)-\mu^{1}\left(\begin{array}{c}
e^{1} \\
0^{2} \\
0 \\
0
\end{array}\right)-\mu^{2}\left(\begin{array}{c}
0^{1} \\
e^{2} \\
0 \\
0
\end{array}\right)=\left(\begin{array}{c}
0^{1} \\
0^{2} \\
1 \\
1
\end{array}\right) .
\end{aligned}
$$


Given the number of equations and unknowns, for each admissible set $B$ a one-dimensional solution set is what one expects. Slightly strengthening the definition of van den Elzen and Talman (1991), we define a bimatrix game to be nondegenerate if at each solution $(t, \sigma, \tau, \lambda, \mu)$ of (6) at most one of the constraints $0 \leq t \leq 1, \lambda_{j}^{1} \geq 0$ for $s_{j}^{1} \notin B, \lambda_{j}^{2} \geq 0$ for $s_{j}^{2} \notin B, \tau_{j}^{1} \geq 0$ fors $s_{j}^{1} \in B$, and $\tau_{j}^{2} \geq 0$ for $s_{j}^{2} \in B$, is binding. This implies that for each $B$ the set of solutions to the corresponding system is an empty set or a compact line segment.

Let $B$ be the set containing for each player $i$ his best response against $v^{-i}$. For nondegenerate games, this best response, and thereby $B$, is uniquely defined. It is easily verified that $t=0, \tau=0, \mu^{i}=u^{i}\left(v^{-i}, s_{j}^{i}\right)$ for $s_{j}^{i} \in B$ and $\lambda_{j}^{i}=\mu^{i}-u^{i}\left(v^{-i}, s_{j}^{i}\right)$, $s_{j}^{i} \in S$, is a solution to (6). Notice that $\lambda_{j}^{i}$ is strictly positive for $s_{j}^{i} \notin B$ and $\tau_{j}^{i}$ is strictly positive for $s_{j}^{i} \in B$.

The idea is to keep $B$ fixed and to increase $t$ until one of the constraints would get violated, i.e. one of the $\lambda$ 's or $\tau$ 's would become negative. Since the set of solutions is a line segment, increasing $t$ leads from the solution just identified, to the other boundary point of the line segment. From a computational point of view, the operation just described corresponds to a linear programming pivot step.

If the other boundary point of the line segment corresponds to $t=1$, the algorithm stops. It is easily verified that a solution to (6) with $t=1$ yields a Nash equilibrium of the bimatrix game $\Gamma$. Otherwise, we have to adapt the set $B$ such that the procedure can be continued. For the first line segment generated, the case where $\tau_{j}^{i}$ becomes negative for some $s_{j}^{i} \in B$ cannot occur. The end-point of the line segment is characterized by $\lambda_{j}^{i}=0$ for some $s_{j}^{i} \notin B$. In this case $s_{j}^{i}$ is added to the set $B$ and the system of equations (6) is studied for $B \cup\left\{s_{j}^{i}\right\}$. The end-point of the previous line segment is the starting point of the line segment of solutions to (6) for $B \cup\left\{s_{j}^{i}\right\}$. The end-point of this line segment satisfies either $t=1$, in which case a Nash equilibrium has been found, or $\tau_{j^{\prime}}^{i^{\prime}}=0$ for some $s_{j^{\prime}}^{i^{\prime}} \in B \cup\left\{s_{j}^{i}\right\}$, in which case a new admissible set is defined as $B \cup\left\{s_{j}^{i}\right\} \backslash\left\{s_{j^{\prime}}^{i^{\prime}}\right\}$, or $\lambda_{j^{\prime}}^{i^{\prime}}=0$ for some $s_{j^{\prime}}^{i^{\prime}} \notin B \cup\left\{s_{j}^{i}\right\}$, and a new admissible set is defined as $B \cup\left\{s_{j}^{i}\right\} \cup\left\{s_{j^{\prime}}^{i^{\prime}}\right\}$.

The procedure stops when $t$ is equal to 1 . The door-in door-out principle of Lemke and Howson guarantees that such will be the case after generating a finite number of admissible sets $B$. Hence starting from a solution corresponding to $(t, \sigma)=(0, v)$ by linear programming steps a piecewise linear path towards a Nash equilibrium is followed.

Theorem 6 Let the bimatrix game $\left(A^{1}, A^{2}\right)$ and the starting point $v$ be nondegenerate. Then the van den Elzen-Talman algorithm terminates with a Nash equilibrium.

Figure 3 displays the projection on $\Sigma$ of the path for the game of Fig. 1 for initial starting profile $v=\left(\left(\frac{4}{5}, \frac{1}{5}\right),\left(\frac{2}{5}, \frac{3}{5}\right)\right)$. For player 1 and 2 , the pure strategies $s_{2}^{1}$ and $s_{2}^{2}$ are the best responses against the initial strategy profile $v$. Therefore we start tracking the solutions to the system in (6) for $B=\left\{s_{2}^{1}, s_{2}^{2}\right\}$ starting at $t=0$. We can increase $t$ until $t$ reaches $\frac{1}{6}$, the strategy profile $\sigma$ equals $\left(\left(\frac{2}{3}, \frac{1}{3}\right),\left(\frac{1}{3}, \frac{2}{3}\right)\right)$, and $\lambda_{1}^{2}$ becomes 0 . This 
Fig. 3 Example van den

Elzen-Talman

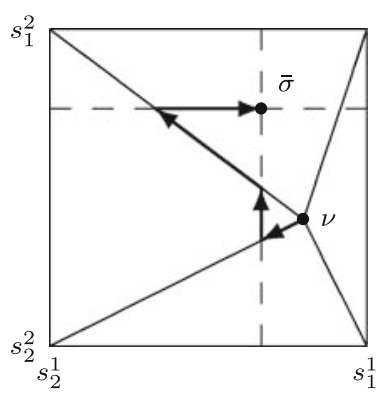

means that at $t=\frac{1}{6}$ the pure strategy $s_{1}^{2}$ has become a best response for player 2 and we should continue tracking the system determined by $B$ extended with $s_{1}^{2}$. In the new system for $B=\left\{s_{2}^{1}, s_{1}^{2}, s_{2}^{2}\right\}$ we can keep $t$ at $\frac{1}{6}$ and increase $\tau_{1}^{2}$ until $\tau_{2}^{2}$ becomes 0 and strategy $s_{2}^{2}$ should be eliminated from the admissible subset $B$. The strategy profile reached equals $\sigma=\left(\left(\frac{2}{3}, \frac{1}{3}\right),\left(\frac{1}{2}, \frac{1}{2}\right)\right)$. The next system to solve is therefore determined by $B=\left\{s_{2}^{1}, s_{1}^{2}\right\}$ and we move in the direction of $\left(s_{2}^{1}, s_{1}^{2}\right)$ by increasing $t, \tau_{2}^{1}$, and $\tau_{1}^{2}$. At $t=\frac{7}{12}, \sigma=\left(\left(\frac{1}{3}, \frac{2}{3}\right),\left(\frac{3}{4}, \frac{1}{4}\right)\right), \lambda_{1}^{1}$ becomes 0 and $s_{1}^{1}$ should be included in $B$ : $B=\left\{s_{1}^{1}, s_{2}^{1}, s_{1}^{2}\right\}$. Next the path stays at $t=\frac{7}{12}$ and $\tau_{1}^{1}$ is increased. We can increase $\tau_{1}^{1}$ until $\tau_{1}^{1}=\frac{1}{3}$ (and $\tau_{2}^{1}=\frac{1}{4}$ ) when $\lambda_{1}^{1}$ gets 0 . The strategy profile $\sigma$ reached equals $\bar{\sigma}=\left(\left(\frac{1}{3}, \frac{2}{3}\right),\left(\frac{3}{4}, \frac{1}{4}\right)\right)$, the Nash equilibrium of the game. We complete the algorithm by following the path for $B=\left\{s_{1}^{1}, s_{2}^{1}, s_{1}^{2}, s_{2}^{2}\right\}$ keeping $\sigma$ at the equilibrium value $\bar{\sigma}$, towards $t=1$.

The procedure tracks a piecewise linear path starting at $(0, v)=\left(0,\left(\left(\frac{4}{5}, \frac{1}{5}\right),\left(\frac{2}{5}\right.\right.\right.$, $\left.\left.\left.\frac{3}{5}\right)\right)\right)$, proceeding to $\left(\frac{1}{6},\left(\left(\frac{2}{3}, \frac{1}{3}\right),\left(\frac{1}{3}, \frac{2}{3}\right)\right)\right),\left(\frac{1}{6},\left(\left(\frac{2}{3}, \frac{1}{3}\right),\left(\frac{1}{2}, \frac{1}{2}\right)\right)\right),\left(\frac{7}{12},\left(\left(\frac{1}{3}, \frac{2}{3}\right),\left(\frac{3}{4}, \frac{1}{4}\right)\right)\right)$, and $\left(\frac{7}{12},\left(\left(\frac{2}{3}, \frac{1}{3}\right),\left(\frac{3}{4}, \frac{1}{4}\right)\right)\right)$, and reaching $t=1$ with the Nash equilibrium at $(1, \bar{\sigma})=$ $\left(1,\left(\left(\frac{2}{3}, \frac{1}{3}\right),\left(\frac{3}{4}, \frac{1}{4}\right)\right)\right)$. Clearly, we could have terminated the algorithm at $t=\frac{7}{12}$ when we found the Nash equilibrium. More generally, as soon as a set $B=S$ is reached, a fully mixed Nash equilibrium has been found, and the algorithm can be stopped.

In van den Elzen and Talman (1999) it is shown that given any prior and a generic bimatrix game, the paths generated by the linear tracing procedure developed in Harsanyi (1975) and the pivoting procedure outlined above are the same. The tracing procedure is the key ingredient in the equilibrium selection method developed in Harsanyi and Selten (1988). We will explain the linear tracing procedure in more detail in Sect. 8.

\section{From two to many players}

The problem of finding a Nash equilibrium in a game with more than two players is equivalent to solving a non-linear complementarity problem. Therefore, complementary pivoting techniques are not directly applicable. For general $n$-person games, algorithms to compute equilibria rely either on simplicial subdivisions for approxi- 
mating nonlinear homotopies by piecewise linear ones, or on numerical methods to solve systems of higher-degree polynomial inequalities. Methods for the computation of equilibria for noncooperative games with more than two players are also surveyed in McKelvey and McLennan (1996).

Seminal papers on the computation of Nash equilibria in $n$-person games are the ones by Rosenmüller (1971) and Wilson (1971). Both extend the LemkeHowson procedure to nondegerenerate $n$-person games by formulating the problem of finding a Nash equilibrium as a nonlinear complementarity problem. Both procedures start with fixing a strategy for $(n-1)$ players and compute the best response against it for the remaining player. The point found determines a starting point for the 2-person game that results after unfixing one of the players from its strategy from which a path towards an equilibrium of this 2-person game originates. This equilibrium determines the starting point for the next step in the procedure. The procedures of Rosenmüller and Wilson construct in this way an equilibrium of an $n$-person game by successively constructing equilibria of $k$-person games, where $1 \leq k \leq n$.

The problem of this procedure is that the paths are no longer linear. To turn this procedure into a numerical algorithm, a method is needed to follow the non-linear path. Although the methods of Rosenmüller and Wilson are not directly suitable for computational purposes, their common result, the existence of a non-linear path leading to an equilibrium, was a very important step towards an implementable algorithm as developed by Garcia et al. (1973) and van der Laan et al. (1987).

A problem of the algorithms discussed so far in this section is that they calculate only an approximation of a sample Nash equilibrium. McLennan (2005) shows that the number of Nash equilibria in normal form games of modest size is huge on average. For instance, the mean number of Nash equilibria in a game with 4 agents, each having 6 strategies, is estimated to be 2,037. This number increases rapidly in the number of players and the number of strategies. The huge number of Nash equilibria of a typical game calls for the development of a theory of equilibrium selection.

Two methods of equilibrium selection stand out in the game-theoretic literature. The first one is the equilibrium selection theory developed in Harsanyi and Selten (1988), which applies to general $n$-person games. The Herings-van den Elzen and Herings-Peeters algorithms compute the Nash equilibrium that is selected by the linear tracing procedure - an important construct in this selection theory. The first of these algorithms relies on simplicial approximations and is explained in detail in Sect. 8. The other algorithm uses tools from differential topology and is presented in Sect. 9.

The second method of equilibrium selection is the quantal response equilibrium of McKelvey and Palfrey (1995). The quantal response equilibrium is often applied by experimentalists because of its good prediction of human behavior. It incorporates probabilities of making mistakes into the Nash equilibrium concept. Quantal response equilibrium can be turned into a theory of equilibrium selection by selecting the equilibrium that is obtained in the limit when mistake probabilities go to zero. This method relies like the Herings-Peeters algorithm on differentiability and is presented in Sect. 10. 


\section{$8 \boldsymbol{n}$-Person games: the Herings-van den Elzen algorithm}

\subsection{The linear tracing procedure}

The linear tracing procedure of Harsanyi (1975) starts from a prior, reflecting the ideas of any player about the strategy used by the other player, and next players react optimally thereupon. Then the players observe that their expectations are not met and adjust them towards observed behavior and again react thereupon. By simultaneously and gradually adjusting expectations, and reacting optimally against these revised expectations, eventually an equilibrium is reached.

Consider some $n$-person game $\Gamma$ and some prior $p \in \Sigma$ and denote, for every $t \in[0,1], \Gamma(t)$ as the game $\left\langle N,\left\{S^{i}\right\}_{i \in N},\left\{v^{i}(t)\right\}_{i \in N}\right\rangle$, where the payoff function $v^{i}(t): \Sigma \rightarrow \mathbb{R}$ of player $i$ is defined by

$$
v^{i}(t, \sigma)=t u^{i}(\sigma)+(1-t) u^{i}\left(p^{-i}, \sigma^{i}\right) .
$$

The game $\Gamma(0)$ corresponds to a trivial game, where all players believe that their opponents play with probability 1 according to the prior belief. The game $\Gamma$ (1) coincides with the original game $\Gamma$. A best response against a strategy combination $\sigma \in \Sigma$ in the game $\Gamma(t)$ corresponds to a best response against the probability distribution $t[\sigma]+(1-t)[p]$ on $S$ in the game $\Gamma$. The latter probability distribution does in general not belong to $\Sigma$, since it may be correlated when there are more than two players.

The set of all Nash equilibria related to the games $\Gamma(t), t \in[0,1]$, is denoted by

$$
\mathcal{L}(\Gamma, p)=\{(t, \sigma) \in[0,1] \times \Sigma \mid \sigma \in \mathrm{NE}(\Gamma(t))\}
$$

Observe that $\sigma \in H(t, \sigma)$ for the homotopy (3) if and only if $(t, \sigma) \in \mathcal{L}(\Gamma, p)$.

Once the number of pure strategies of each player is fixed, a noncooperative game is completely determined by the utility functions $u$, which can be represented by a vector in $\mathbb{R}^{m^{*} \cdot n}$, where $m^{*}$ equals the product over $i$ of $m^{i}$. The standard topology and measure on $\mathbb{R}^{m^{*} \cdot n}$ therefore induce a topology and a measure on games. The set of such games is denoted $\mathcal{G}$. For the following result, see Schanuel et al. (1991), Herings (2000), and Herings and Peeters (2001).

Theorem 7 There exists a path in $\mathcal{L}(\Gamma, p)$ connecting a best response against the prior to a Nash equilibrium of the game $\Gamma$. For an open set of games $\Gamma \in \mathcal{G}$ and priors $p \in \Sigma$ with full Lebesgue measure, this path is unique.

The first of part of Theorem 7 follows from an immediate application of Theorem 2. We refer the reader to Sect. 9 for details on the proof of the second part of Theorem 7.

The linear tracing procedure links a Nash equilibrium of the game $\Gamma(0)$ to a Nash equilibrium of $\Gamma(1)$. That is, the linear tracing procedure traces the generically unique path in $\mathcal{L}(\Gamma, p)$. The interpretation of the linear tracing procedure is that players gradually adjust their beliefs about the behavior of their opponents by gradually putting less weight to the initial beliefs, the prior. 
Recall that a subset $B$ of $S$ is said to be admissible if $B \cap S^{i} \neq \emptyset$ for all $i \in N$. Admissible subsets $B$ can be used to decompose $\mathcal{L}(\Gamma, p)$ in subsets $\mathcal{L}(\Gamma, p, B)$, where a set $\mathcal{L}(\Gamma, p, B)$ contains those elements of $\mathcal{L}(\Gamma, p)$ where only strategies in $B$ are played with positive probability. The manifold structure of $\mathcal{L}(\Gamma, p, B)$ is analyzed in detail in Sect. 9. The set $\Sigma(B)=\left\{\sigma \in \Sigma \mid \forall s_{j}^{i} \notin B, \sigma_{j}^{i}=0\right\}$ is the strategy set consistent with $B$. The set $\Lambda(B)=\left\{\lambda \in \mathbb{R}_{+}^{m} \mid \forall s_{j}^{i} \in B, \lambda_{j}^{i}=0\right\}$ is the set of Lagrange multipliers associated to $B$. A point $(t, \sigma) \in[0,1] \times \Sigma(B)$ belongs to $\mathcal{L}(\Gamma, p, B)$ if and only if there exists $\lambda \in \Lambda(B)$ and $\mu \in \mathbb{R}^{n}$ such that

$$
v^{i}\left(t, \sigma^{-j}, s_{j}^{i}\right)+\lambda_{j}^{i}-\mu^{i}=0 \quad\left(s_{j}^{i} \in S\right) .
$$

We denote by $v$ the function with components $v^{i}\left(t, \sigma^{-j}, s_{j}^{i}\right)$ for $s_{j}^{i} \in S$. The next step is to turn Theorem 7 and Eq. (7) into an implementable algorithm.

In Herings and van den Elzen (2002) a simplicial algorithm is used to generate a piecewise linear path that approximates $\mathcal{L}(\Gamma, p)$. For every $B$, they define a piecewise linear approximation of (7), whose solution approximates $\mathcal{L}(\Gamma, p, B)$. Next it is shown that the piecewise linear solutions paths for varying $B$ can be nicely fitted together, and result in a piecewise linear path from a solution to the starting problem to an approximate Nash equilibrium of the terminal problem.

\subsection{Piecewise linear approximations}

This subsection describes how to construct piecewise linear approximations to the function $v$.

For $k \in \mathbb{N}$, a $k$-dimensional simplex or $k$-simplex $\varsigma$ in $\mathbb{R}^{d}$ is defined as the convex hull of $k+1$ affinely independent points $x^{1}, \ldots, x^{k+1}$ of $\mathbb{R}^{d}$. We write $\varsigma=\varsigma\left(x^{1}, \ldots, x^{k+1}\right)$ and call $x^{1}, \ldots, x^{k+1}$ the vertices of $\varsigma$. A $(k-1)$-simplex that is the convex hull of $k$ vertices of $\varsigma\left(x^{1}, \ldots, x^{k+1}\right)$ is said to be a facet of $\zeta$. The facet $\tau\left(x^{1}, \ldots, x^{j-1}, x^{j+1}, \ldots, x^{k+1}\right)$ is called the facet of $\varsigma$ opposite to the vertex $x^{j}$. For a non-negative integer $k^{\prime}$ less than or equal to $k$, a $k^{\prime}$-simplex that is the convex hull of $k^{\prime}+1$ vertices of $\varsigma$ is said to be a $k^{\prime}$-face of $\varsigma$.

A finite collection $\mathcal{S}$ of $k$-simplices is a triangulation of a $k$-dimensional convex subset $T$ of $\mathbb{R}^{d}$ if (1) $T$ is the union of all simplices in $\mathcal{S}$ and (2) the intersection of any two simplices in $\mathcal{S}$ is either empty or a common face of both. If $\mathcal{S}$ is a triangulation of $T$, and a facet $\tau$ of $\varsigma^{1} \in \mathcal{S}$ is a subset of the relative boundary of $T$, then there is no $\varsigma^{2} \in \mathcal{S}$ such that $\varsigma^{2} \neq \varsigma^{1}$ and $\tau$ is a facet of $\varsigma^{2}$. If $\tau$ is not a subset of the relative boundary of $T$, then there is exactly one $\varsigma^{2} \in \Sigma$ such that $\varsigma^{2} \neq \varsigma^{1}$ and $\tau$ is also a facet of $\varsigma^{2}$. The mesh size of a triangulation $\mathcal{S}$ of $T$ is defined by $\operatorname{mesh}(\mathcal{S})=\max \left\{\|x-y\|_{\infty} \mid x, y \in \varsigma, \varsigma \in \mathcal{S}\right\}$.

It is well-known that full-dimensional affine parts of the relative boundary of a set are triangulated by the facets of the simplices in a triangulation. More precisely, let $\mathcal{S}$ be a triangulation of a convex $k$-dimensional subset $T$ of $\mathbb{R}^{d}$, and let the $(k-1)$ dimensional subset $\bar{T}$ of the relative boundary of $T$ be such that $\bar{T}$ is equal to the affine hull of $\bar{T}$ intersected with $T$. Then the collection $\overline{\mathcal{S}}$ given by $\overline{\mathcal{S}}=\{\tau \subset \bar{T} \mid \exists \varsigma \in \mathcal{S}, \tau$ is a facet of $\varsigma\}$ is a triangulation of $\bar{T}$ (see Todd (1976), Theorem 2.3). For instance, 


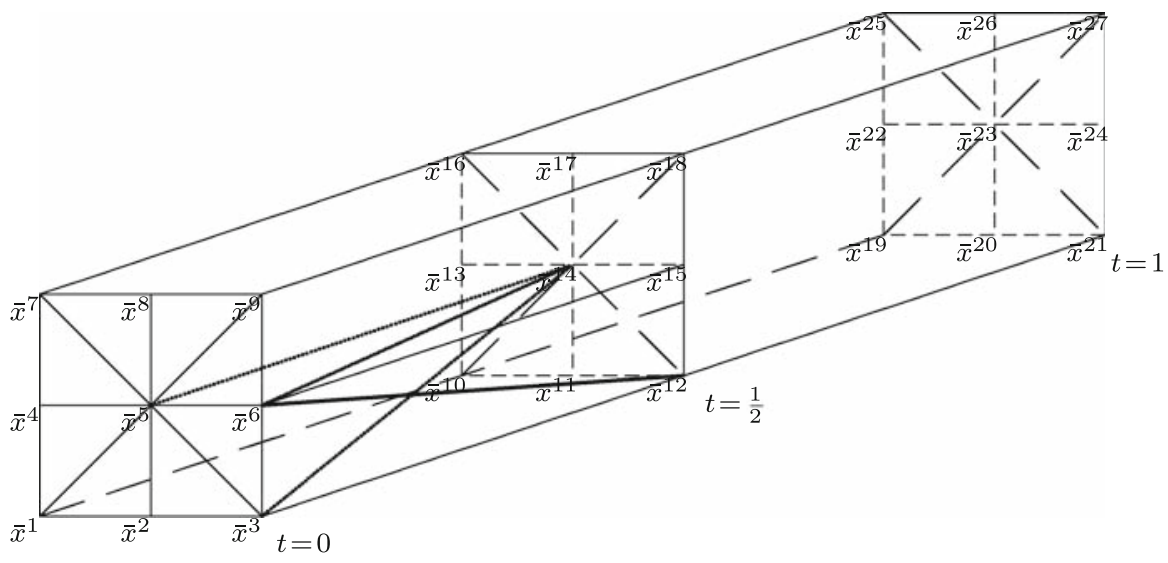

Fig. 4 A triangulation of $[0,1] \times \Sigma$. Only 3 -simplices in $\operatorname{co}\left(\bar{x}^{3}, \bar{x}^{5}, \bar{x}^{6}, \bar{x}^{12}, \bar{x}^{14}, \bar{x}^{15}\right)$ are depicted. The vertex $\bar{x}^{1}=(0,(1,0),(1,0))$ refers to the strategy vector at which both players play their first pure strategy. Similarly, $\bar{x}^{3}, \bar{x}^{7}$, and $\bar{x}^{9}$ correspond to $(0,(0,1),(1,0)),(0,(1,0),(0,1))$, and $(0,(0,1),(0,1))$, respectively

Table 2 All full-dimensional simplices in $\left[0, \frac{1}{2}\right] \times S$

\begin{tabular}{llll}
\hline $\operatorname{co}\left(\bar{x}^{4}, \bar{x}^{7}, \bar{x}^{5}, \bar{x}^{14}\right)$ & $\operatorname{co}\left(\bar{x}^{8}, \bar{x}^{7}, \bar{x}^{5}, \bar{x}^{14}\right)$ & $\operatorname{co}\left(\bar{x}^{8}, \bar{x}^{9}, \bar{x}^{5}, \bar{x}^{14}\right)$ & $\operatorname{co}\left(\bar{x}^{6}, \bar{x}^{9}, \bar{x}^{5}, \bar{x}^{14}\right)$ \\
$\operatorname{co}\left(\bar{x}^{4}, \bar{x}^{7}, \bar{x}^{16}, \bar{x}^{14}\right)$ & $\operatorname{co}\left(\bar{x}^{8}, \bar{x}^{7}, \bar{x}^{16}, \bar{x}^{14}\right)$ & $\operatorname{co}\left(\bar{x}^{8}, \bar{x}^{9}, \bar{x}^{18}, \bar{x}^{14}\right)$ & $\operatorname{co}\left(\bar{x}^{6}, \bar{x}^{9}, \bar{x}^{18}, \bar{x}^{14}\right)$ \\
$\operatorname{co}\left(\bar{x}^{4}, \bar{x}^{13}, \bar{x}^{16}, \bar{x}^{14}\right)$ & $\operatorname{co}\left(\bar{x}^{8}, \bar{x}^{17}, \bar{x}^{16}, \bar{x}^{14}\right)$ & $\operatorname{co}\left(\bar{x}^{8}, \bar{x}^{17}, \bar{x}^{18}, \bar{x}^{14}\right)$ & $\operatorname{co}\left(\bar{x}^{6}, \bar{x}^{15}, \bar{x}^{18}, \bar{x}^{14}\right)$ \\
$\operatorname{co}\left(\bar{x}^{4}, \bar{x}^{1}, \bar{x}^{5}, \bar{x}^{14}\right)$ & $\operatorname{co}\left(\bar{x}^{2}, \bar{x}^{1}, \bar{x}^{5}, \bar{x}^{14}\right)$ & $\operatorname{co}\left(\bar{x}^{2}, \bar{x}^{3}, \bar{x}^{5}, \bar{x}^{14}\right)$ & $\operatorname{co}\left(\bar{x}^{6}, \bar{x}^{3}, \bar{x}^{5}, \bar{x}^{14}\right)$ \\
$\operatorname{co}\left(\bar{x}^{4}, \bar{x}^{1}, \bar{x}^{10}, \bar{x}^{14}\right)$ & $\operatorname{co}\left(\bar{x}^{2}, \bar{x}^{1}, \bar{x}^{10}, \bar{x}^{14}\right)$ & $\operatorname{co}\left(\bar{x}^{2}, \bar{x}^{3}, \bar{x}^{12}, \bar{x}^{14}\right)$ & $\operatorname{co}\left(\bar{x}^{6}, \bar{x}^{3}, \bar{x}^{12}, \bar{x}^{14}\right)$ \\
$\operatorname{co}\left(\bar{x}^{4}, \bar{x}^{13}, \bar{x}^{10}, \bar{x}^{14}\right)$ & $\operatorname{co}\left(\bar{x}^{2}, \bar{x}^{11}, \bar{x}^{10}, \bar{x}^{14}\right)$ & $\operatorname{co}\left(\bar{x}^{2}, \bar{x}^{11}, \bar{x}^{12}, \bar{x}^{14}\right)$ & $\operatorname{co}\left(\bar{x}^{6}, \bar{x}^{15}, \bar{x}^{12}, \bar{x}^{14}\right)$ \\
\hline
\end{tabular}

the set $\{0\} \times \Sigma$ is triangulated by the facets of the simplices in a triangulation of $[0,1] \times \Sigma$.

An example of a triangulation of $[0,1] \times \Sigma$ is illustrated in Fig. 4 for the case we have two players each having two pure strategies. For later purposes we give all 3-simplices in the triangulation of Fig. 4 in Table 2. In Table 2 only the 3 -simplices in $\left[0, \frac{1}{2}\right] \times \Sigma$ are given. The ones in $\left[\frac{1}{2}, 1\right] \times \Sigma$ follow by means of a translation. The position in the table is related to the position of a simplex in the triangulation.

A function $\bar{v}:[0,1] \times \Sigma \rightarrow \mathbb{R}^{n}$ is called the piecewise linear approximation of $v$ with respect to $\mathcal{S}$ if for each vertex $x^{k}$ of any $\varsigma\left(x^{1}, \ldots, x^{m-n+2}\right) \in \mathcal{S}, \bar{v}\left(x^{k}\right)=v\left(x^{k}\right)$ and $\bar{v}$ is affine on each simplex of $\mathcal{S}$. Hence, if $x$ belongs to $\varsigma\left(x^{1}, \ldots, x^{m-n+2}\right)$, that is $x=\sum_{k=1}^{m-n+2} \alpha_{k} x^{k}$ where $\alpha_{k} \geq 0, k=1, \ldots, m-n+2$ and $\sum_{k=1}^{m-n+2} \alpha_{k}=1$, then $\bar{v}(x)=\sum_{k=1}^{m-n+2} \alpha_{k} \bar{v}\left(x^{k}\right)$.

Let an admissible subset $B$ and a triangulation $\mathcal{S}$ of $[0,1] \times \Sigma$ be given. We denote by $\mathcal{S}(B)$ the collection of $b$-faces of simplices in $\Sigma$ that are contained in $[0,1] \times \Sigma(B)$, where $b=|B|+1-n$ with $|B|$ the cardinality of $B$. By repeated application of the 
result that claims that the relative boundary of a set is triangulated by the facets of a triangulation, it follows that $\mathcal{S}(B)$ is a triangulation of $[0,1] \times \Sigma(B)$.

Let a simplex $\varsigma\left(x^{1}, \ldots, x^{b+1}\right) \in \mathcal{S}(B)$ be given. Consider solutions $(\alpha, \lambda, \mu)$ in $\mathbb{R}_{+}^{b+1} \times \Lambda(B) \times \mathbb{R}^{n}$ of the following system of equations, a piecewise linear approximation of the system (7),

$$
\begin{gathered}
\sum_{k=1}^{b+1} \alpha_{k}=1 \\
\sum_{k=1}^{b+1} \alpha_{k} \bar{v}^{i}\left(x^{k}\right)+\sum_{s_{j}^{i} \notin B} \lambda_{j}^{i} e_{j}^{i}-\mu^{i} e^{i}=0^{i} \quad(i \in N),
\end{gathered}
$$

where we observe that $\bar{v}^{i}\left(x^{k}\right)=v^{i}\left(x^{k}\right)$. Such solutions are called admissible. An admissible solution $(\alpha, \lambda, \mu)$ to (8) corresponds to an approximate Nash equilibrium $\sigma$ of $\Gamma(t)$, where $\left(t, \sigma^{\top}\right)^{\top}=\sum_{k=1}^{b+1} \alpha_{k} x^{k}$. Indeed, strategies in $B$ are best replies for the payoff function $\bar{v}, \lambda_{j}^{i}$ is the payoff gap between strategy $s_{j}^{i}$ and a best reply for player $i$, and $\mu^{i}$ is the payoff for player $i$ according to $\bar{v}$ when he uses a best reply. Since $\zeta \subset[0,1] \times \Sigma(B)$, strategies that are not a best reply are played with probability zero. An admissible solution to (8) is said to be degenerate if at least two of the variables $\alpha_{k}, k=1, \ldots, b+1$, and $\lambda_{j}^{i}, s_{j}^{i} \notin B$, are equal to zero.

\subsection{Complete facets}

The Herings-van den Elzen algorithm generates by means of lexicographic pivoting techniques a piecewise linear path of approximate Nash equilibria in $[0,1] \times \Sigma$ joining $\{0\} \times \Sigma$ to $\{1\} \times \Sigma$. The path is such that every $(t, \sigma)$ on it corresponds to an admissible $B$, a simplex $\varsigma \in \mathcal{S}(B)$, and an admissible solution $(\alpha, \lambda, \mu)$. The Herings-van den Elzen algorithm specifies in a unique way how to move from one simplex to another.

For given $(B, \varsigma),(8)$ corresponds to a linear system with $m+1$ equations and $m+2$ variables. If we rule out degeneracies, then a non-empty solution set is a onedimensional compact line segment. The end-points of the line segment are either approximate Nash equilibria for $\Gamma(0)$ or $\Gamma(1)$, or yield admissible solutions for a new $(\bar{B}, \bar{\zeta})$. Indeed, with degeneracies ruled out, at an end-point either $\alpha_{k}=0$ for exactly one $k$ or $\lambda_{j}^{i}=0$ for exactly one $s_{j}^{i} \notin B$. In the first case, the end-point belongs to the facet $\tau$ of $\varsigma$ opposite to the vertex $x^{k}$. If $\tau$ does not belong to the relative boundary of $[0,1] \times \Sigma(B)$, then there is a unique simplex $\bar{\zeta} \in \mathcal{S}(B)$ such that $\bar{\zeta} \neq \varsigma$, and $\tau$ is a facet of $\bar{\zeta}$. The algorithm continues by generating a line-segment of admissible solutions in $\bar{\zeta}$. If $\tau$ belongs to the relative boundary of $[0,1] \times \Sigma(B)$, then the endpoint is either an approximate Nash equilibrium for $\Gamma(0)$, or an approximate Nash equilibrium for $\Gamma(1)$, or $\tau \in \mathcal{S}(\bar{B})$, with $\bar{B}$ a uniquely determined subset of $B$ having one element less, and the algorithm continues with a line-segment of admissible solutions in $\tau$. If $\lambda_{j}^{i}=0$, then also strategy $s_{j}^{i}$ is a best reply. The algorithm continues 
with a line-segment of admissible solutions in $\bar{\zeta}$, where $\bar{\zeta}$ is the unique simplex in $\mathcal{S}\left(B \cup\left\{s_{j}^{i}\right\}\right)$ having $\varsigma$ as a facet.

We have argued in Sect. 5 that degeneracy is not always a non-generic phenomenon in game theory. Degeneracy can be dealt with by exploiting lexicographic pivoting techniques. We explain next how lexicographic pivoting techniques can be used to extend the ideas of the previous paragraph to handle degenerate games.

For an admissible $B$ and a facet $\tau\left(x^{1}, \ldots, x^{b}\right)$ of a simplex in $\mathcal{S}(B)$, the $(m+1) \times$ $(m+1)$-matrix $A_{B, \tau}$ is defined by

$$
A_{B, \tau}=\left[\begin{array}{ccccccccc}
1 & \cdots & 1 & 0 & \cdots & \cdots & \cdots & \cdots & 0 \\
& & & E^{1} & & 0 & -e^{1} & & 0 \\
\bar{v}\left(x^{1}\right) & \cdots & \bar{v}\left(x^{b}\right) & 0 & \ddots & 0 & 0 & \ddots & 0 \\
& & & 0 & 0 & E^{n} & 0 & 0 & -e^{n}
\end{array}\right],
$$

where, for $i \in N, E^{i}=\left[e_{j}^{i}\right]_{s_{j}^{i} \notin B}$, and the zeros indicate submatrices of appropriate dimension with zeros only. The matrix $A_{B, \tau}$ corresponds to the coefficients in (8) when a facet $\tau$ of a simplex $\varsigma$ is considered. Suppose $A_{B, \tau}^{-1}$ exists. From $A_{B, \tau} A_{B, \tau}^{-1}=I^{m+1}$ (the $(m+1)$-dimensional identity matrix), it follows that the first column of $A_{B, \tau}^{-1}$ corresponds to an admissible solution to (8) for any $\varsigma \in \mathcal{S}(B)$ being the convex hull of $\tau$ and some vertex $x^{b+1} \in[0,1] \times \Sigma(B)$, whenever the first $m+1-n$ components of this column are non-negative. No restrictions are imposed on the last $n$ rows of $A_{B, \tau}^{-1}$. In a nondegenerate solution the first $m+1-n$ components are all strictly positive, since $\alpha_{b+1}=0$ extends the admissible solution for the facet $\tau$ to the simplex $\varsigma$.

A row vector $x \in \mathbb{R}^{m+1}$ is lexicographically positive if it is not equal to the vector of zeroes and its first non-zero entry is positive. The matrix $A_{B, \tau}^{-1}$ is said to be semi-lexicopositive if each of the first $m+1-n$ rows is lexicographically positive.

Given a linear system of equations as in (8), one can pivot in a uniquely determined new column, either of the type $\left(1, \bar{v}\left(x^{b+1}\right)\right)$ or of the type $\left(0,0, e_{j}^{i}, 0\right)$. In the nondegenerate case such a pivot step determines in a unique way a column out of the first $m+1-n$ to be replaced. In degenerate cases it is possible that the leaving column is not uniquely determined. A semi-lexicographic pivot step is a pivot step where the leaving column is selected in such a way that the inverse of the resulting matrix $A_{B, \tau}$ is semi-lexicopositive. Herings and van den Elzen (2002) show that a semi-lexicographic pivot step determines in all cases a unique column out of the first $m+1-n$ to be replaced.

A facet $\tau$ of a simplex in $\mathcal{S}(B)$ is $B$-complete if $A_{B, \tau}^{-1}$ exists and is semi-lexicopositive. Theorem 8 describes all possible cases that may occur if a $B$-complete facet $\tau$ is given and a semi-lexicographic pivot step with a vector $\left(1, \bar{v}\left(x^{b+1}\right)\right)$ is made, where $x^{b+1}$ is a vertex of a simplex having $\tau$ as the facet opposite to it.

Theorem 8 Let $(\Gamma, p, \mathcal{S})$ and a $B$-complete facet $\tau$ of a simplex $\varsigma \in \mathcal{S}(B)$ be given. Then exactly one of the following cases holds: 
1. 5 has exactly one other $B$-complete facet $\bar{\tau}$,

2. $\checkmark$ is $\bar{B}$-complete for precisely one admissible $\bar{B}$.

Theorem 9 describes all possible cases that may occur if a $B$-complete facet $\tau$ is given that is also a simplex belonging to $\mathcal{S}(\bar{B})$, where $B=\bar{B} \cup\left\{s_{j}^{i}\right\}$, and a semilexicographic pivot step with a vector $\left(0,0, e_{j}^{i}, 0\right)$ is made.

Theorem 9 Let $(\Gamma, p, \mathcal{S})$ and a $B$-complete facet $\tau$ that belongs to $\mathcal{S}(\bar{B})$ for some admissible $\bar{B}$ be given. Then exactly one of the following cases holds:

1. $\tau$ is $\widehat{B}$-complete for precisely one admissible $\widehat{B}$ with $\widehat{B} \neq B$,

2. precisely one facet $v$ of $\tau$ is $\bar{B}$-complete.

The consideration of $B$-complete facets determines a unique starting point for the algorithm. The admissible subset $B^{0}$ is defined by the set of strategies $s_{j}^{i}$, where $j$ is the largest integer such that $s_{j}^{i}$ is a best reply to the prior $p$ for player $i$. Notice that $\left|B^{0}\right|=n$. It can be shown that the facet (vertex) $\tau=\{0\} \times \Sigma\left(B^{0}\right)$ is $B^{0}$-complete and that there is no other $B$-complete facet $\tau$ in $\{0\} \times \Sigma$. Even in degenerate cases, the semi-lexicographic rules single out the unique $B^{0}$-complete facet $\{0\} \times \Sigma\left(B^{0}\right)$, which serves as a unique starting point of the algorithm.

Notice that we are now in a position that makes the Lemke-Howson door-in doorout principle applicable. There is a unique starting point in $\{0\} \times \Sigma$, the $B^{0}$-complete facet $\{0\} \times \Sigma\left(B^{0}\right)$. For any $B$-complete facet $\tau$ of a simplex in $\mathcal{S}(B)$, Theorems 8 and 9 determine another complete facet in a unique way. The finiteness of the number of simplices guarantees that at some stage a facet that is a subset of $\{1\} \times \Sigma$ is reached. Such a facet determines an approximate Nash equilibrium.

\subsection{The algorithm}

The formal steps of the Herings-van den Elzen algorithm are as follows. In the description of the algorithm the $r$-th $B$-complete facet generated by the algorithm is denoted by $\tau^{r}, r=1,2, \ldots$.

Algorithm Let $(\Gamma, p, \mathcal{S})$ be given.

STEP 0 . Let $b=1$ and $r=1$. Let $B=B^{0}, \tau^{1}=\{0\} \times \Sigma(B)$, and let $x^{2}$ be the unique vertex of the 1 -simplex of $\mathcal{S}(B)$ containing $\tau^{1}$ as the facet opposite to it.

STEP 1. Let $\varsigma$ be equal to the convex hull of $\tau^{r}$ and $\left\{x^{b+1}\right\}$. Make a semi-lexicographic pivot step with $\left(1, \bar{v}\left(x^{b+1}\right)\right)$ into the system of equations (8) corresponding to $A_{B, \tau^{r}}$, yielding a unique column $k^{\prime}$ of $A_{B, \tau^{r}}$ which has to be replaced. If $k^{\prime} \in\{b+1, \ldots, m+1-n\}$, then go to Step 3 with $s_{j^{\prime}}^{i^{\prime}}$ the pure strategy corresponding to column $k^{\prime}$. Otherwise, go to Step 2 .

STEP 2. Increase the value of $r$ by 1 and let $\tau^{r}$ be the facet of $\varsigma$ opposite $x^{k^{\prime}}$. If $\tau^{r} \subset\{1\} \times \Sigma$, then the algorithm terminates with an approximate Nash equilibrium $\bar{\sigma}$ of $\Gamma(1)$ induced by the admissible solution of (8) corresponding to $A_{B, \tau^{r}}$. If $\tau^{r} \in \mathcal{S}(\bar{B})$ for some admissible $\bar{B}$, then go to Step 4. Otherwise, 
there is exactly one $b$-simplex $\bar{\zeta}$ of $\mathcal{S}(B)$ such that $\bar{\zeta} \neq \varsigma$ and $\tau^{r}$ is a facet of $\bar{\zeta}$. Go to Step 1 with $x^{b+1}$ as the unique vertex of $\bar{\zeta}$ opposite $\tau^{r}$.

STEP 3. Let the admissible set $\bar{B}$ be defined by $\bar{B}=B \cup\left\{s_{j^{\prime}}^{i^{\prime}}\right\}$. There is a unique simplex $\bar{\zeta}$ of $\mathcal{S}(\bar{B})$ having $\zeta$ as a facet. Increase the value of both $b$ and $r$ by 1 and go to Step 1 with $x^{b+1}$ as the unique vertex of $\bar{\zeta}$ opposite $\varsigma, B=\bar{B}$, and $\tau^{r}=\varsigma$.

STEP 4. Let $\varsigma$ be equal to $\tau^{r}$. Make a semi-lexicographic pivot step with $\left(0,0, e_{j^{\prime}}^{i^{\prime}}, 0\right)$ into the system of Equations (8) corresponding to $A_{B, \tau^{r}}$, where $s_{j^{\prime}}^{i^{\prime}}$ is such that $\bar{B} \cup\left\{s_{j^{\prime}}^{i^{\prime}}\right\}=B$. This yields a unique column $k^{\prime}$ of $A_{B, \tau^{r}}$ which has to be replaced. If $k^{\prime} \in\{b+1, \ldots, m+1-n\}$, then decrease the value of both $b$ and $r$ by 1 and go to Step 3 with $s_{j^{\prime}}^{i^{\prime}}$ the pure strategy corresponding to column $k^{\prime}$ and $B=\bar{B}$. Otherwise, decrease the value of $b$ by 1 and go to Step 2 with $B=\bar{B}$.

Theorem 8 corresponds to the semi-lexicographic pivot step made in Step 1: Case 1 occurs if one goes from Step 1 to Step 2, and Case 2 if one goes from Step 1 to Step 3. Theorem 9 corresponds to the semi-lexicographic pivot step performed in Step 4: Case 1 happens if one goes from Step 4 to Step 3, and Case 2 if one goes from Step 4 to Step 2. The algorithm terminates after a finite number of steps, after having generated a $B$-complete facet $\tau$ being a subset of $\{1\} \times \Sigma(B)$.

Because we are dealing with a non-linear system of equations, we have to study approximations of Nash equilibria. Our ultimate aim is to compute an $\varepsilon$-Nash equilibrium of $\Gamma$ with $\varepsilon$ an arbitrarily chosen positive number. For $\varepsilon \geq 0$, a mixed strategy combination $\sigma \in \Sigma$ is called an $\varepsilon$-Nash equilibrium of $\Gamma$ if, for every $i \in N, \sigma_{k}^{i}>0$ implies $u^{i}\left(\sigma^{-i}, s_{k}^{i}\right) \geq \max _{s_{j}^{i} \in S^{i}} u^{i}\left(\sigma^{-i}, s_{j}^{i}\right)-\varepsilon$. In an $\varepsilon$-Nash equilibrium the loss in payoffs of using a suboptimal strategy is at most $\varepsilon$. Though the suboptimal strategy itself might be far away from an optimal strategy, the loss in payoff is small, which makes sense from a game-theoretic standpoint.

The algorithm generates a piecewise linear approximation of the homotopy path generated by the linear tracing procedure. For every $\varepsilon>0$, there is $\delta>0$ such that a triangulation of $[0,1] \times \Sigma$ with mesh size smaller than $\delta$ makes the piecewise linear approximation generated by the Herings-van den Elzen algorithm within $\varepsilon$-Hausdorff distance from the homotopy path generated by the tracing procedure and hence the algorithm terminates with an $\varepsilon$-Nash equilibrium of $\Gamma$. If the latter path is unique, and a sequence of triangulations with decreasing mesh size is taken, then the generated piecewise linear approximations converge to the tracing procedure path in the Hausdorff topology. As a consequence, the induced sequence of $\varepsilon$-Nash equilibria converges to the Nash equilibrium selected by the tracing procedure.

\subsection{An example}

In Fig. 5 the algorithm is illustrated for the game considered before. The prior $p$ is taken to be $\left(\left(\frac{1}{2}, \frac{1}{2}\right),\left(\frac{3}{4}, \frac{1}{4}\right)\right)$. Since $p^{2}$ is part of the Nash equilibrium, both pure strategies of player 1 are best responses, and this game is degenerate both in the sense of 


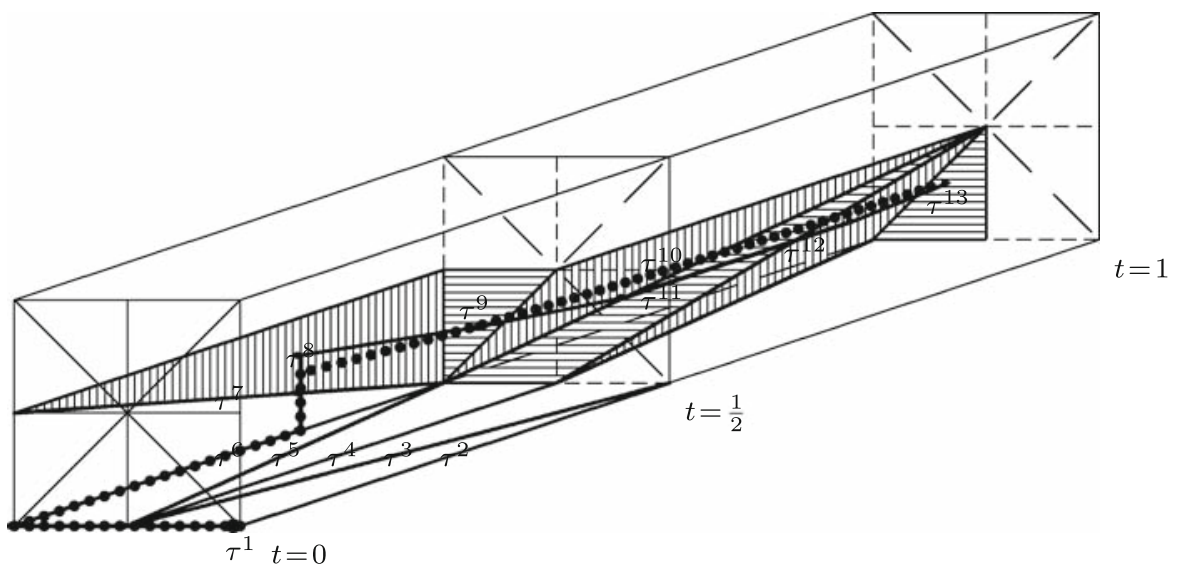

Fig. 5 The Herings-van den Elzen algorithm in action

van den Elzen-Talman and in the sense of Herings-van den Elzen. We have chosen this prior to illustrate that lexicographic pivoting techniques overcome degeneracies. Since the game is a bimatrix game, we would in general recommend to use either the Lemke-Howson or the van den Elzen-Talman algorithm, rather than the Herings-van den Elzen algorithm.

In the example the algorithm generates 13 facets before terminating with an approximate Nash equilibrium. The facet $\tau^{1}$ is zero-dimensional, the facets $\tau^{2}, \ldots, \tau^{7}$ are one-dimensional, and the facets $\tau^{8}, \ldots, \tau^{13}$ are two-dimensional. The two-dimensional facets are shaded in Fig. 5. The path generated by the algorithm is illustrated by the heavily drawn line going from $\tau^{1}$ to $\tau^{13}$. The dotted line represents $\mathcal{L}(\Gamma, p)$, which for this example consists of one component. It can be decomposed into six sets $\mathcal{L}(\Gamma, p, B)$.

\section{9 -Person games: the Herings-Peeters algorithm}

\subsection{Some tools from differential topology}

Games with $n$ players possess a nice piecewise differentiable structure that can be exploited for computational purposes. To prove Theorem 12, the theory of regular constraint sets as presented in Jongen et al. (1983) can be applied, see also Herings (1997) for a first application of this theory to economics.

For some $r \geq 1$ a subset $M$ of $\mathbb{R}^{d}$ is called a $k$-dimensional $C^{r}$ manifold with generalized boundary (MGB), if for every $\bar{x} \in M$ there exists a $C^{r}$ diffeomorphism $\varphi: U \rightarrow V$, where $U$ is an open subset of $\mathbb{R}^{d}$ containing $\bar{x}$ and $V$ is open in $\mathbb{R}^{d}$, and some integer $\ell(\bar{x}) \geq 0$, such that $\varphi(\bar{x})=0$ and $\varphi(U \cap M)$ equals $\left\{y \in V \mid y_{l}=0, l=\right.$ $1, \ldots, d-k$, and $\left.y_{l} \geq 0, l=d-k+1, \ldots, d-k+\ell(\bar{x})\right\}$. If for every element $\bar{x}$ of an MGB $M$ it holds that $\ell(\bar{x}) \leq 1$, then $M$ is called a manifold with boundary and the set of elements $\bar{x}$ for which $\ell(\bar{x})=1$ is an $(k-1)$-dimensional manifold, called the boundary of $M$. One way to show that a set is an MGB is by showing that it is a regular constraint set, a concept introduced next. 
Let $K^{1}$ and $K^{2}$ be two finite index sets and let $g_{k}$ for all $k \in K^{1}$ and $h_{k}$ for all $k \in K^{2}$, be $C^{r}$ functions defined on some open subset $X$ of $\mathbb{R}^{d}$. We define

$$
M[g, h]=\left\{x \in X \mid g_{k}(x)=0, \forall k \in K^{1} \text {, and } h_{k}(x) \geq 0, \forall k \in K^{2}\right\} .
$$

For $x \in X$ we define the set $K^{0}(x)=\left\{k \in K^{2} \mid h_{k}(x)=0\right\}$. If for every $\bar{x} \in M[g, h]$ it holds that

$$
\left\{\partial_{x} g_{k}(\bar{x}) \mid k \in K^{1}\right\} \cup\left\{\partial_{x} h_{k}(\bar{x}) \mid k \in K^{0}(\bar{x})\right\}
$$

is a set of linearly independent vectors, then $M[g, h]$ is called a $C^{r}$ regular constraint set (RCS). In Jongen et al. (1983) it is shown that every $C^{r} \operatorname{RCS}$ is an $\left(d-\left|K^{1}\right|\right)$ dimensional $C^{r}$ MGB with $\ell(\bar{x})=\left|K^{0}(\bar{x})\right|$ for every $\bar{x} \in M[g, h]$. If a set is a $k$-dimensional manifold with generalized boundary, then the neighborhood of a point $\bar{x}$ belonging to that set looks, in a well-defined sense, like $\mathbb{R}^{k-\ell(\bar{x})} \times \mathbb{R}_{+}^{\ell(\bar{x})}$.

Let $C^{1}$ manifolds $X, Y$, and $Z, Z$ being a subset of $Y$, an element $\bar{x}$ of $X$, and a function $f \in C^{1}(X, Y)$ be given. The function $f$ is said to intersect $Z$ transversally at $\bar{x} \in X$, denoted by $f \mp Z$ at $\bar{x}$, if

$$
f(\bar{x}) \notin Z \text {, or } f(\bar{x}) \in Z \text { and } T_{f(\bar{x})} Z+\partial f(\bar{x})\left(T_{\bar{x}} X\right)=T_{f(\bar{x})} Y,
$$

where $T_{\bar{x}} X$ denotes the tangent space of $X$ at $\bar{x}$. For regular constraint sets, the tangent space of $X$ at $\bar{x}$ is easily computed as

$$
T_{\bar{x}} X=\left\{x \in \mathbb{R}^{d} \mid \partial g(\bar{x})(x)=0\right\},
$$

where 0 is an $(d-k)$-dimensional vector of zeroes with $k$ being the dimension of the tangent space. The function $f$ is said to intersect $Z$ transversally if $f 历 Z$ at every $x \in X$. Transversality of $f$ implies that the inverse image of $f$ has a particularly nice structure.

Theorem 10 For $k^{1}, k^{2}, k^{3} \in\{0\} \cup \mathbb{N}$ and $r \in \mathbb{N} \cup\{\infty\}$, let a $k^{1}$-dimensional $C^{r}$ manifold $X$, a $k^{2}$-dimensional $C^{r}$ manifold $Y$, and a $k^{3}$-dimensional $C^{r}$ manifold $Z$, $Z$ being a subset of $Y$, be given. Moreover, let $f \in C^{r}(X, Y)$ be a function such that $f$ Z. If $k^{1}-k^{2}+k^{3} \geq 0$, then $f^{-1}(Z)$ is a $\left(k^{1}-k^{2}+k^{3}\right)$-dimensional $C^{r}$ manifold. If $k^{1}-k^{2}+k^{3}<0$, then $f^{-1}(Z)=\emptyset$.

We are now in a position to state the transversality theorem [see, for instance, Mas-Colell (1985)].

Theorem 11 For $k^{1}, k^{2}, k^{3} \in\{0\} \cup \mathbb{N}$ and $r \in \mathbb{N} \cup\{\infty\}$ with $r \geq \max \left(\left\{1, k^{1}-\right.\right.$ $\left.k^{2}+k^{3}\right\}$ ), let a $k^{1}$-dimensional $C^{r}$ manifold $X$, a $k^{2}$-dimensional $C^{r}$ manifold $Y, a$ $k^{3}$-dimensional $C^{r}$ manifold $Z, Z$ being a subset of $Y$, be given. Moreover, let a $C^{r}$ manifold $\Omega$ and a function $f \in C^{r}(X \times \Omega, Y)$ be given. For every $\omega \in \Omega$, define a function $f^{\omega} \in C^{r}(X, Y)$ by $f^{\omega}(x)=f(x, \omega), \forall x \in X$. Then $f$ $\bar{Z}$ implies $f^{\omega} \bar{\hbar} Z$, except for a subset of $\Omega$ having Lebesgue measure zero in $\Omega$. 
9.2 The manifold structure of the linear tracing procedure

The Herings-Peeters algorithm exploits the manifold structure of the sets $\mathcal{L}(\Gamma, p, B)$. They prove the following result.

Theorem 12 For an open set of games $\Gamma \in \mathcal{G}$ and priors $p \in \Sigma$ with full Lebesgue measure, for all admissible subsets $B$ of $S$, the set $\mathcal{L}(\Gamma, p, B)$ is a compact onedimensional $C^{\infty}$ manifold with boundary. Moreover, $(t, \sigma)$ is a boundary point of $\mathcal{L}(\Gamma, p, B)$ if and only if either $\sigma_{j}^{i}=0$ for exactly one $s_{j}^{i} \in B$, or exactly one $s_{j}^{i} \notin B$ is a best response to $\sigma^{-i}$, or $t=0$, or $t=1$.

A compact one-dimensional $C^{\infty}$ manifold with boundary consists of finitely many arcs and loops. Theorem 12 therefore guarantees that generically $\mathcal{L}(\Gamma, p, B)$ has a simple mathematical structure, excluding bifurcations, spirals, higher dimensional parts, etc. Theorem 12 is the non-linear equivalent of the compact line segments of the Lemke-Howson, van den Elzen-Talman, and van den Elzen-Herings algorithms. One difference now is that loops are possible and, moreover, $\mathcal{L}(\Gamma, p, B)$ may contain more than one arc or loop.

We can show $\mathcal{L}(\Gamma, p, B)$ to be a $C^{\infty}$ one-dimensional manifold with boundary, if we can represent it as a $C^{\infty}$ regular constraint set with index sets $K^{1}$ and $K^{2}$, functions $g_{k}$ for $k \in K^{1}$ holding with equality, and functions $h_{k}$ for $k \in K^{2}$ holding with weak inequality, where $\left|K^{1}\right|=d-1$. Moreover, we have to show that $\left|K^{0}(\bar{x})\right| \leq 1$ for every $\bar{x} \in M[g, h]$.

A point $(t, \sigma)$ belongs to $\mathcal{L}(\Gamma, p, B)$ if and only if there exists $\lambda \in \mathbb{R}^{m}$ and $\mu \in \mathbb{R}^{n}$ such that

$$
\begin{array}{ll}
v^{i}\left(t, \sigma^{-i}, s_{j}^{i}\right)+\lambda_{j}^{i}-\mu^{i}=0 & \left(s_{j}^{i} \in S\right) \\
\sigma_{j}^{i}=0 & \left(s_{j}^{i} \notin B\right) \\
\lambda_{j}^{i}=0 & \left(s_{j}^{i} \in B\right) \\
\sum_{s_{j}^{i} \in S^{i}} \sigma_{j}^{i}-1=0 & (i \in N) \\
\sigma_{j}^{i} \geq 0 & \left(s_{j}^{i} \in B\right) \\
\lambda_{j}^{i} \geq 0 & \left(s_{j}^{i} \notin B\right) \\
t \geq 0 & \\
-t+1 \geq 0 . &
\end{array}
$$

The set $K^{1}$ corresponding to (10) has cardinality $2 m+n$. This is indeed one less than the number of free variables, which is equal to $2 m+n+1$. Unfortunately, (10) is not a regular constraint set in general. The set corresponding to (9) may not consist of independent vectors. Moreover, it is easy to construct examples such that $\left|K^{0}(\bar{x})\right| \geq 2$ for some $\bar{x} \in M[g, h]$. Fortunately, such examples can be shown to be exceptional cases. To prove that, the transversality theorem comes to rescue. 
We apply Theorem 11 in the following way. We take as the set of parameters $\Omega$ the set of games, so $\Omega=\mathbb{R}^{m^{*} \cdot n}$. Then we specify three types of systems of equations where variables belong to a Euclidean space with appropriate dimension. The first type consists of all equalities of (10), the second type of all equalities plus one inequality of (10) formulated as an equality, and the third type of all equalities plus two inequalities of (10) formulated as equalities. Thus we obtain three types of functions $f$, with variables $t, \sigma, \lambda, \mu, \omega$, that can be shown to satisfy $f$ 雨 $\{0\}$, and as a consequence $f^{\omega} \pitchfork\{0\}$ for almost every $\omega \in \Omega$. Counting the number of equations and unknowns, using Theorem 10, it follows that a function $f^{\omega}$ of the third type, with two inequalities, can only be transversal if $f^{\omega^{-1}}\{0\}=\emptyset$. From this we derive the conclusion that, generically, it is impossible that two inequality constraints are binding at the same time. The transversality of the first two types of functions leads to the conclusion that, generically, (10) is a regular constraint set. This concludes the basic steps in the proof of Theorem 12.

Using the representation of $\mathcal{L}(\Gamma, p, B)$ as a regular constraint set, we also find that a point $(t, \sigma)$ is a boundary point of $\mathcal{L}(\Gamma, p, B)$ if and only if either $\sigma_{j}^{i}=0$ for exactly one $s_{j}^{i} \in B$ or exactly one $s_{j}^{i} \notin B$ is a best response to $\sigma^{-i}$, or $t=0$, or $t=1$. Moreover, two sets $\mathcal{L}(\Gamma, p, B)$ and $\mathcal{L}(\Gamma, p, \bar{B})$ can only have boundary points in common, since otherwise (10) would have a solution with two inequality constraints binding at the same time. When there is a common boundary point, there is an optimal strategy $s_{j}^{i}$ that is played with zero probability, with the two admissible subsets only differing in this strategy: $(B \cup \bar{B}) \backslash(B \cap \bar{B})=\left\{s_{j}^{i}\right\}$. Moreover, in each point at most two sets can meet, as we would again obtain a case where (10) has two inequality constraints binding at the same time. We see that all subsets $\mathcal{L}(\Gamma, p, B)$ of $\mathcal{L}(\Gamma, p)$ are nicely connected. What we have obtained is a full non-linear analogue of the door-in door-out principle of Lemke-Howson.

Theorem 13 For an open set of games $\Gamma \in \mathcal{G}$ and priors $p \in \Sigma$ with full Lebesgue measure, $\mathcal{L}(\Gamma, p)$ is a compact one-dimensional piecewise $C^{\infty}$ manifold with boundary. All boundary points of $\mathcal{L}(\Gamma, p)$ are in $\{0,1\} \times \Sigma$. The boundary point in $\{0\} \times \Sigma$ is unique.

For almost every $\Gamma$ and $p$, the set $\mathcal{L}(\Gamma, p)$ consists of a finite number of paths and loops. Although it is not necessarily the case that these paths and loops are smooth, the number of non-differentiabilities is finite at most. All paths in $\mathcal{L}(\Gamma, p)$ start and end in $\{0,1\} \times \Sigma$. Each such path consists of a finite sequence of smooth arcs of the sets $\mathcal{L}(\Gamma, p, B)$. A loop in $\mathcal{L}(\Gamma, p)$ consists either of a finite sequence (at least two) of differentiable arcs in the sets $\mathcal{L}(\Gamma, p, B)$ or is a loop of one set $\mathcal{L}(\Gamma, p, B)$.

Since $\mathcal{L}(\Gamma, p)$ consists of finitely many one-dimensional $C^{\infty}$ manifolds with boundary, it is possible to use standardized software based on predictor-corrector methods, to approximately follow each manifold. We refer the interested reader to Allgower and Georg (1990) for a detailed exposition on predictor-corrector methods. 
9.3 Smoothening the linear tracing procedure

It is attractive to avoid switching from one system of equations to the next, and to formulate one, differentiable, system of equations whose solution corresponds to $\mathcal{L}(\Gamma, p)$. This is the purpose of the current section.

Define, for $\alpha \in \mathbb{R}^{m}$, functions $\sigma: \mathbb{R}^{m} \rightarrow \mathbb{R}^{m}$ and $\lambda: \mathbb{R}^{m} \rightarrow \mathbb{R}^{m}$ by $\sigma_{j}^{i}(\alpha)=$ $\left[\max \left\{0, \alpha_{j}^{i}\right\}\right]^{2}$ and $\lambda_{j}^{i}(\alpha)=\left[\max \left\{0,-\alpha_{j}^{i}\right\}\right]^{2}$. Next consider the system

$$
\begin{aligned}
& v^{i}\left(t, \sigma^{-i}(\alpha), s_{j}^{i}\right)+\lambda_{j}^{i}(\alpha)-\mu^{i}=0 \quad\left(s_{j}^{i} \in S\right) \\
& \sum_{s_{j}^{i} \in S^{i}} \sigma_{j}^{i}(\alpha)-1=0 \quad(i \in N) \\
& t \geq 0 \\
& -t+1 \geq 0 .
\end{aligned}
$$

For each point $(t, \alpha, \mu)$ satisfying the (in)equalities (11), the point $(t, \sigma(\alpha), \lambda(\alpha), \mu)$ satisfies the (in)equalities (10) with $B=\left\{s_{j}^{i} \in S \mid \sigma_{j}^{i}(\alpha)>0\right\}$.

Let $\mathcal{H}:[0,1] \times \mathbb{R}^{m} \times \mathbb{R}^{n} \rightarrow \mathbb{R}^{m} \times \mathbb{R}^{n}$ be the continuously differentiable homotopy defined by

$$
\mathcal{H}(t, \alpha, \mu)=\left(\begin{array}{cc}
v^{i}\left(t, \sigma^{-i}(\alpha), s_{j}^{i}\right)+\lambda_{j}^{i}(\alpha)-\mu^{i} & \left(s_{j}^{i} \in S^{i}, i \in N\right) \\
\sum_{s_{j}^{i} \in S^{i}} \sigma_{j}^{i}(\alpha)-1 & (i \in N)
\end{array}\right) .
$$

The zeros of this homotopy describe the linear tracing procedure: $(t, \alpha, \mu) \in \mathcal{H}^{-1}(\{0\})$ if and only if $(t, \sigma(\alpha)) \in \mathcal{L}(\Gamma, p)$. For a generic game and prior, there is a unique point $\left(0, \alpha^{0}, \mu^{0}\right) \in \mathcal{H}^{-1}(\{0\})$ at $t=0$ at which our algorithm starts. Following the path described by the zeros of $\mathcal{H}$, we end up in a point $(1, \tilde{\alpha}, \tilde{\mu}) \in \mathcal{H}^{-1}(\{0\})$. This point generates the Nash equilibrium $\sigma(\tilde{\alpha})$ of $\Gamma$ selected by the Harsanyi-Selten theory.

Theorem 14 For an open set of games $\Gamma \in \mathcal{G}$ and priors $p \in \Sigma$ with full Lebesgue measure, $\mathcal{H}^{-1}(\{0\})$ is a compact one-dimensional $C^{1}$ manifold with boundary. All boundary points of $\mathcal{H}^{-1}(\{0\})$ are in $\{0,1\} \times \mathbb{R}^{m} \times \mathbb{R}^{n}$. The boundary point in $\{0\} \times \mathbb{R}^{m} \times \mathbb{R}^{n}$ is unique.

The set $\mathcal{H}^{-1}(\{0\})$ consists of finitely many differentiable arcs and loops. All arcs start and end in $\{0,1\} \times \mathbb{R}^{m} \times \mathbb{R}^{n}$. Loops have no points in common with $\{0,1\} \times$ $\mathbb{R}^{m} \times \mathbb{R}^{n}$. There is exactly one arc that starts in $\{0\} \times \mathbb{R}^{m} \times \mathbb{R}^{n}$ and that ends in $\{1\} \times \mathbb{R}^{m} \times \mathbb{R}^{n}$ with a point $(1, \tilde{\alpha}, \tilde{\mu})$ that generates the Nash equilibrium selected by the tracing procedure. This arc is a transformation of the feasible path of the tracing procedure. All other arcs start and end in $\{1\} \times \mathbb{R}^{m} \times \mathbb{R}^{n}$ and connect two points inducing Nash equilibria of $\Gamma$.

The structure of $\mathcal{H}^{-1}(\{0\})$ is even simpler than the one of $\mathcal{L}(\Gamma, p)$. Not only, like for $\mathcal{L}(\Gamma, p)$, are complications like bifurcations, spirals, higher dimensional solutions sets, diverging behavior, etc. excluded. The arcs and loops in $\mathcal{H}^{-1}(\{0\})$ are differentiable everywhere. It is the transformation of variables, via the functions $\sigma(\cdot)$ and 
$\lambda(\cdot)$ that smoothes out the kinks. As a direct consequence, it is possible to calculate the derivative at each point of the feasible path, which makes it possible to follow the path by means of differentiable as opposed to simplicial methods. In Herings and Peeters (2001) we report numerical results using the software-package HOMPACK, a FORTRAN77 program. This program implements three predictor-corrector methods, see Watson et al. (1987) for an introduction to HOMPACK.

\subsection{An example}

For the game in Fig. 1 and as prior the mixed strategy combination $p=\left(\left(\frac{1}{2}, \frac{1}{2}\right)\right.$, $\left.\left(\frac{1}{2}, \frac{1}{2}\right)\right)$, the corresponding set $\mathcal{L}(\Gamma, p)$ is given by

$$
\begin{aligned}
\mathcal{L}(\Gamma, p) & =\left\{\left(t,\left(s_{2}^{1}, s_{1}^{2}\right)\right) \mid t \in\left[0, \frac{1}{2}\right]\right\} \quad \cup \quad\left\{\left(\frac{1}{2},\left((r, 1-r), s_{1}^{2}\right)\right) \mid r \in\left[0, \frac{5}{6}\right]\right\} \\
& \cup\left\{\left(t,\left(\left(\frac{3 t+1}{6 t}, \frac{3 t-1}{6 t}\right),\left(\frac{2 t+1}{4 t}, \frac{2 t-1}{4 t}\right)\right)\right) \mid t \in\left[\frac{1}{2}, 1\right]\right\} .
\end{aligned}
$$

At $t=0$, for players 1 and 2 , the strategies $s_{2}^{1}$ and $s_{1}^{2}$ respectively are the best responses against the prior. The first part of the path of $\mathcal{L}(\Gamma, p)$ is therefore determined by $\mathcal{L}(\Gamma, p, B)$ for $B=\left\{s_{2}^{1}, s_{1}^{2}\right\}$. As long as $t \leq \frac{1}{2}$ the path stays at $\left(s_{2}^{1}, s_{1}^{2}\right)$. At $t=\frac{1}{2}$ strategy $s_{1}^{1}$ of player 1 becomes optimal and has to be added to the admissible set $B$ such that the path continues in $\mathcal{L}(\Gamma, p, B)$ for $B=\left\{s_{1}^{1}, s_{2}^{1}, s_{1}^{2}\right\}$. In this new component, at $t=\frac{1}{2}$ the weight on player 1's strategy $s_{1}^{1}$ is increased until either it gets weight 1 or until player 2's unused strategy $s_{2}^{2}$ becomes optimal. The latter happens when the weight on strategy $s_{1}^{1}$ reaches the value $\frac{5}{6}$. The final part of the path is determined by the component $\mathcal{L}(\Gamma, p, B)$ with $B$ including all strategies.

The first plot of Fig. 6 shows the values of $t, \sigma$, and $\lambda$ along the homotopy path that is generated by the linear tracing procedure as a function of pathlength $\tau$. To make the plot more clear, only informative values of the variables are depicted. In particular this means that for each pure strategy $s_{j}^{i}$, either $\sigma_{j}^{i}$ or $-\lambda_{j}^{i}$ is plotted, depending on which one is non-zero. We see that at two values of $\tau$ kinks in the paths occur. These values of $\tau$ correspond to the two points where we had to adapt the admissible subset $B$ in the description above.

The second plot shows the values of $t$ and the coordinates of $\alpha$ as a function of pathlength and therefore corresponds to the feasible path of the tracing procedure after application of the transformation of variables. From the plot it is seen that the transformation indeed causes the kinks in the path to disappear. At the points where previously kinks occurred, now the derivatives of all variables with respect to pathlength are equal to zero, except the variable that passes zero at that point. It is precisely the transformation of variables that does the trick. If the pathlength $\tau$ is interpreted as the speed of path tracking, the transformation causes a delay in speed at points where kinks occur in the original path. 

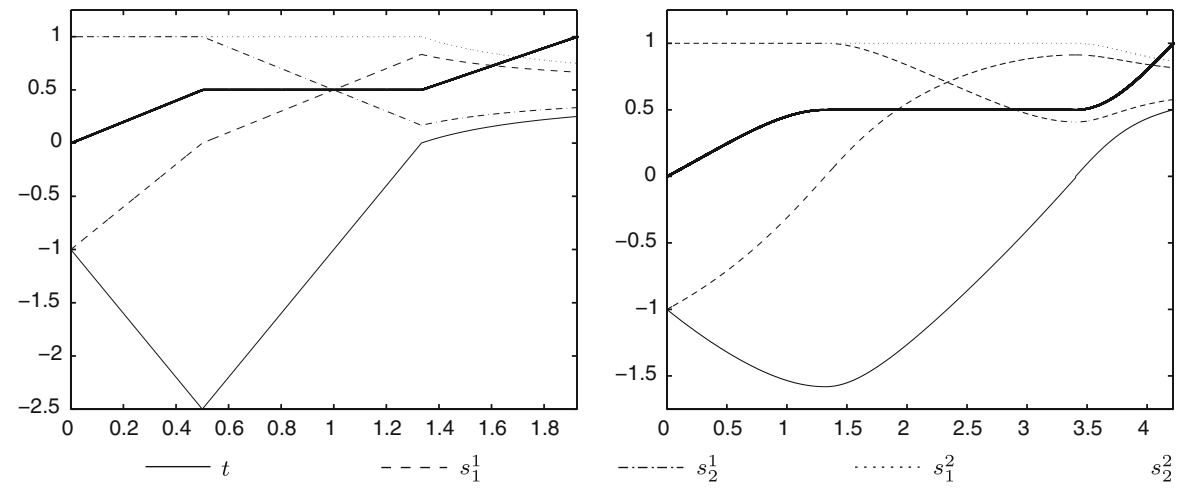

Fig. 6 The homotopy path before and after transformation

\section{$10 n$-Person games: the McKelvey-Palfrey algorithm}

Quantal response equilibria as introduced by McKelvey and Palfrey (1995) are statistical versions of Nash equilibria where each player's payoff is subject to random error. For a given specification of the error structure, a quantal response equilibrium is a mixed strategy combination that is consistent with optimizing behavior subject to that error structure. One possible interpretation of this is that players make calculation errors according to some random process when calculating their expected payoffs.

Player $i$ 's payoff when playing pure strategy $s_{j}^{i}$ against a mixed strategy combination $\sigma^{-i}$ is subject to error and is given by

$$
\hat{u}^{i}\left(\sigma^{-i}, s_{j}^{i}\right)=u^{i}\left(\sigma^{-i}, s_{j}^{i}\right)+\varepsilon_{j}^{i} .
$$

We define $\varepsilon^{i}=\left(\varepsilon_{j}^{i}\right)_{s_{j}^{i} \in S^{i}}$ and $\varepsilon=\left(\varepsilon^{i}\right)_{i \in N}$. We assume that $\varepsilon$ is determined by an absolutely continuously distributed random variable $\underline{\varepsilon}$ with density function $\varphi$. Moreover, the random variables $\underline{\varepsilon}^{i}$ are independent and the expected value of each random variable $\underline{\varepsilon}_{j}^{i}$ exists.

For any possible opponents' mixed strategy combination $\sigma^{-i}$, the $s_{j}^{i}$-response set $R_{j}^{i}$ is defined as the set of error vectors that make strategy $s_{j}^{i}$ the best response, so

$$
R_{j}^{i}\left(\sigma^{-i}\right)=\left\{\varepsilon^{i} \mid \hat{u}^{i}\left(\sigma^{-i}, s_{j}^{i}\right) \geq \hat{u}^{i}\left(\sigma^{-i}, s_{k}^{i}\right) \text { for all } s_{k}^{i} \in S^{i}\right\} .
$$

The probability of choosing pure strategy $s_{j}^{i}$ is then given by

$$
\pi_{j}^{i}\left(\sigma^{-i}\right)=\int_{R_{j}^{i}\left(\sigma^{-i}\right)} \varphi^{i}\left(\varepsilon^{i}\right) \mathrm{d} \varepsilon^{i} .
$$

The function $\pi^{i}$ is called the statistical reaction function, or the quantal response function and satisfies the feature that better strategies are more likely to be chosen than 
worse strategies. A quantal response equilibrium (QRE) is a mixed strategy combination $\sigma$ that is consistent with the error structure:

$$
\sigma_{j}^{i}=\pi_{j}^{i}\left(\sigma^{-i}\right) \text { for all } s_{j}^{i} \in S^{i} \text { and all } i \in N .
$$

For the following result, see McKelvey and Palfrey (1995).

Theorem 15 For any game $\Gamma$ and for any density function $\varphi$, a QRE exists.

For any parameter $\lambda \geq 0$, the logistic quantal response function is defined by

$$
\pi_{j}^{i}\left(\sigma^{-i}\right)=\frac{\exp \left(\lambda u^{i}\left(\sigma^{-i}, s_{j}^{i}\right)\right)}{\sum_{s_{k}^{i} \in S^{i}} \exp \left(\lambda u^{i}\left(\sigma^{-i}, s_{k}^{i}\right)\right)}
$$

and is obtained as statistical reaction function when $\varphi^{i}$ corresponds to the extreme value (or log-Weibull) distribution. Therefore, if each player uses a logistic quantal response function, the corresponding QRE, called logit equilibrium, requires that

$$
\sigma_{j}^{i}=\frac{\exp \left(\lambda u^{i}\left(\sigma^{-i}, s_{j}^{i}\right)\right)}{\sum_{s_{k}^{i} \in S^{i}} \exp \left(\lambda u^{i}\left(\sigma^{-i}, s_{k}^{i}\right)\right)} \quad \text { for all } s_{j}^{i} \in S^{i} \text { and all } i \in N \text {. }
$$

The parameter $\lambda$ is inversely related to the error level. When $\lambda=0$, the choice of the players is completely determined by the errors which induces all players to play all their pure strategies with equal probability. When $\lambda$ approaches infinity, the influence of the error disappears.

The logit equilibrium correspondence $\mathcal{L}: \mathbb{R}_{+} \rightarrow \Sigma$ is given by

$$
\mathcal{L}(\lambda)=\left\{\sigma \in \Sigma \mid \sigma_{j}^{i}=\frac{\exp \left(\lambda u^{i}\left(\sigma^{-i}, s_{j}^{i}\right)\right)}{\sum_{s_{k}^{i} \in S^{i}} \exp \left(\lambda u^{i}\left(\sigma^{-i}, s_{k}^{i}\right)\right)} \quad\left(s_{j}^{i} \in S^{i}, i \in N\right)\right\} .
$$

For the following result, see McKelvey and Palfrey (1995).

Theorem 16 When $\lambda$ approaches infinity, the set of logit equilibria converges to a subset of the set of Nash equilibria. For an open set of games $\Gamma \in \mathcal{G}$ with full Lebesgue measure, the graph of $\mathcal{L}$ contains a unique path of logit equilibria that starts at the centroid for $\lambda=0$ and converges to a unique Nash equilibrium as $\lambda$ goes to infinity.

The latter unique Nash equilibrium is called the limiting logit equilibrium of the game $\Gamma$. This induces a unique selection from the set of Nash equilibria by 'tracing' the graph of the logit equilibrium correspondence beginning at the centroid of the mixed strategy simplex and continuing for increasing values of $\lambda$.

We define the correspondence $\widetilde{\mathcal{L}}:[0,1] \rightarrow \Sigma$ by

$$
\widetilde{\mathcal{L}}(t)= \begin{cases}\mathcal{L}\left(\frac{t}{1-t}\right) & \text { if } t \in[0,1) \\ \mathrm{NE}(\Gamma) & \text { if } t=1 .\end{cases}
$$

For the following result, see Herings (2002). 
Theorem 17 For all games $\Gamma \in \mathcal{G}$ the graph of $\widetilde{\mathcal{L}}$ contains a component containing an element at $t=0$ and at $t=1$.

The (homotopy) path of logit equilibria can be traced from the centroid at $t=0$ towards the limiting logit equilibrium at $t=1$ using one of the two methods described in the previous two sections. Turocy (2005) describes a technique to efficiently trace the unique branch generated by the logit equilibrium correspondence by exploiting convergence properties of the logit QRE.

\section{$11 n$-Person games: other algorithms}

Govindan and Wilson (2003) provide a generalization of the Lemke-Howson algorithm to $n$-person games. They indicate that one of the implications of the structure theorem of Kohlberg and Mertens (1986) is that, above each generic ray emanating from the game of interest (represented as a point in a Euclidean space), the graph of the equilibrium correspondence is a one-dimensional manifold. ${ }^{3}$ Moreover, at sufficient distance from the relevant game there is a unique equilibrium. Therefore, starting from a sufficiently distant game along any generic ray, one can traverse the line segment to the relevant game, tracing the one-dimensional manifold of equilibria along the way, to find an equilibrium of the game of interest at the terminus. Govindan and Wilson (2003) propose to trace the manifold using a global Newton method. For bimatrix games the Lemke-Howson and van den Elzen-Talman algorithms can be understood as special cases of the procedure of Govindan and Wilson (2003). For the Lemke-Howson algorithm this is also immediate from the exposition in Sect. 4.1. As is shown in Balthasar (2009), also the linear tracing procedure for $n$-person games can be formulated in terms of payoff perturbations corresponding to a generic ray rather than our approach that is in terms of strategy perturbations. Govindan and Wilson (2004) iteratively use polymatrix approximations to increase the speed of their algorithm presented in Govindan and Wilson (2003) by exploiting the presence of linearities.

Another notable equilibrium selection method that has a bounded rationality interpretation is the $t$ - solution (Rosenthal (1989)). Voorneveld (2006) showed that for every game the set of $t$ - solutions constitutes a homotopy path starting at $t=0$ and terminating in a Nash equilibrium at $t=1$. A nice feature of this selection theory is that along the homotopy path players eliminate successively higher levels of neverbest replies and eventually play only rationalizable strategies with positive probability (see Voorneveld (2006)). This path can be followed using the techniques presented in the next two sections.

Yamamoto (1993) presents a homotopy to compute a proper Nash equilibrium. Yamamoto makes use of an expanding set to define a homotopy whose homotopypath connects the centroid of the simplotope to a solution of a stationary point problem that is a Nash equilibrium of the original game.

\footnotetext{
3 Such a result can also be obtained by applying the tools from differential topology explained in Sect. 9.1.
} 


\section{Extensive form and dynamic games}

Since extensive form games can be represented as a game in normal form, in principle it is possible to apply any of the algorithms of the previous sections. There are two caveats. First, since the normal form representation of an extensive form game is non-generic, it becomes crucial to handle degeneracies appropriately. Second, from a computational point of view, it might be much more attractive to work with the extensive form representation where the data structure is relatively more concise. Here, we will restrict ourselves to a brief overview of this important stream of the literature.

Wilson (1972) applied the Lemke-Howson algorithm to a two-person extensive form game. By making use of the extensive form directly, the developed method never deals with the entire linear complementary problem, and leads to significant savings in storage.

In the same journal issue, von Stengel (1996) and Koller et al. (1996) propose algorithms to solve two-person extensive form games: the first for the class of such games with zero-sum payoff structure, the latter for general payoffs. Both papers study the sequence form, rather than the normal form, of the extensive form game. The advantage of the sequence form in comparison to the normal form is that the size of the sequence form is linear and not exponentially in the size of the game tree. For the resulting problem the algorithm of Lemke (1965) is applied.

von Stengel et al. (2002) present another algorithm for solving two-person extensive form games with perfect recall. Just like von Stengel (1996) and Koller et al. (1996), the sequence form is used rather than the normal form, but instead of applying Lemke's algorithm, the van den Elzen-Talman algorithm is used. The advantages of that method are the potential to find multiple equilibria and to find normal form perfect equilibria.

McKelvey and Palfrey (1998) extend the (logit) quantal response equilibrium defined for normal form games to the (logit) agent quantal response equilibrium (AQRE) for extensive form games. In an AQRE, at each information set players choose better strategies with higher probabilities than worse strategies. They show that limit points of the logit AQRE yield a refinement of sequential equilibria for any finite extensive form game, but are not logically related to other refinement criteria such as the intuitive criterion or trembling hand perfection.

For stochastic games, homotopy based algorithms have been proposed by Filar and Raghavan (1984), Nowak and Raghavan (1993), and Raghavan and Syed (2002). All papers concentrate on two-person games with the single-controller property, i.e. only one player has partial control over the state transitions: the first for discounted games with zero-sum payoff structure, the second for discounted games with general payoff structure, the third for undiscounted games. In all papers a stationary equilibrium is defined as a solution to a linear complementary problem, where linearity is obtained as a result of the single-controller property. Subsequently, Lemke's (1965) algorithm is applied to solve for a stationary equilibrium.

Herings and Peeters (2004) extend the linear tracing procedure to a related procedure that is suitable for general finite discounted stochastic games. By application of the technique presented in Sect. 9, the extension is shown to possess the same geomet- 
ric properties as the linear tracing procedure for normal form games. By numerically following the exact homotopy path, which is shown to be generically unique, an approximation of a stationary equilibrium results. Since this method allows for an arbitrary starting point, it has the potential to find multiple stationary equilibria.

\section{Computing all Nash equilibria}

For some purposes, an algorithm to compute a single equilibrium may be insufficient. Even if the algorithm is able to compute an equilibrium that satisfies perfectness or some other refinement criterion, it cannot be ruled out that there might exist another equilibrium that is more salient. For some equilibrium selection theories comparison of equilibria is needed, such as payoff dominance or risk dominance. Moreover, multiple equilibria with different implications (in the comparative statics) may exist. We therefore have an interest in algorithms to compute all equilibria. In the same issue of this journal, Avis et al. (2009) survey algorithms for finding all Nash equilibria of a bimatrix game.

Homotopy-based algorithms that are developed to solve for all Nash equilibria are developed in Kostreva and Kinard (1991) and Herings and Peeters (2005). Both methods are based on the application of numerical techniques to obtain all the solutions to a system of polynomial equations. Kostreva and Kinard (1991) focuses on solving for polynomial optimization problems in general and bimatrix games in particular. Herings and Peeters (2005) concentrates on solving $n$-person noncooperative games for all its Nash equilibria. The proposed method is shown to be globally convergent for an open set of games with full Lebesgue measure.

Datta (2009) presents an algorithm for the computation of all Nash equilibria in generic games. Starting from a specially structured factorizable system the algorithm uses polyhedral homotopy continuation to solve for the equilibria. The Gröbner basis (that is also used in Herings and Peeters (2005)) is applied to gain more geometric information about how the solution set varies with the payoff function.

Recent developments in solving systems of polynomial equations are likely to result in improvements in homotopy methods to compute all Nash equilibria, as well as to new insights in issues concerning the number of Nash equilibria in a game. For a state-of-the-art account of research in systems of polynomial equations, as well as an application to game theory, see Sturmfels (2002).

We have already stressed that even games of modest size may have an enormous number of Nash equilibria. To compute all Nash equilibria of an arbitrary game of moderate size is therefore not feasible within reasonable time limits. The problem is alleviated somewhat since the algorithms described above generate more and more Nash equilibria during their execution. There is no need to wait for the algorithm to terminate before the first output can be observed. Furthermore, it is possible to apply parallel computing to speed up computations.

Also, many games coming from economic applications are not arbitrary, but have a particular structure that may even lead to uniqueness of equilibrium. Instead of an algorithm that determines all Nash equilibria of the game of interest, the economist may be satisfied with an answer to the question whether a particular game has a unique equilib- 
rium or not. Unfortunately, even the much more modest problem of deciding whether a game has a unique equilibrium or not is NP-complete (Gilboa and Zemel 1989).

An alternative uniqueness test consists of running the van den Elzen-Talman algorithm for bimatrix games or the Herings-Peeters algorithm for $n$-person games for a variety of starting points. If the algorithm returns the same equilibrium irrespective of the starting point, then, though perhaps not unique, the equilibrium found has at least a very large basin of attraction. If, by varying the starting point, a second equilibrium is found, then it is also possible to find a third one, as long as the game is nondegenerate. The reason for this is that homotopy algorithms always terminate in a positively indexed equilibrium. A homotopy determines a path from the starting point towards one equilibrium, and connects the remaining equilibria in a pairwise manner. Each connected pair consist of a positively and a negatively indexed equilibrium. If a second equilibrium is found, then it is possible to find a third one, by following the path that connects the first equilibrium to another one.

\section{Conclusions}

The paper presents an overview of homotopy algorithms as applied to non-cooperative game theory. Advantages of homotopy algorithms include their numerical stability, their ability to locate multiple solutions, and the insight they provide in the properties of solutions. Homotopy algorithms can be implemented easily with the aid of existing, professionally programmed, software.

From an algorithmic point of view, it is useful to distinguish bimatrix games from games with more than two players. The reason is that the problem of finding a Nash equilibrium in a bimatrix games is equivalent to solving a linear complementarity problem, whereas the general problem is equivalent to solving a non-linear complementarity problem, and has to rely on numerical approximation techniques.

We present two algorithms in detail that are suitable to compute Nash equilibria for bimatrix games, the Lemke-Howson algorithm and the van den Elzen-Talman algorithm. We argue that both algorithms are best understood as following a solution path generated by a homotopy. We also explain how the door-in door-out principle of Lemke-Howson applies to both algorithms.

For general $n$-person games, it is usually not possible to compute an equilibrium exactly, which calls for different methods. We present the two main ideas in detail, one using simplicial methods, the other predictor-corrector methods. The Herings-van den Elzen algorithm relies on the simplicial approach, the Herings-Peeters algorithm on the predictor-corrector idea. Both algorithms converge to an approximate Nash equilibrium for general $n$-person games. For both methods we also illustrate how they can be understood as following a solution path generated by a homotopy. We also explain how the door-in door-out principle of Lemke-Howson applies to both algorithms. The third method we present to find a Nash equilibrium of a general $n$-person game is related to the quantal-response equilibrium of McKelvey and Palfrey.

Since the number of Nash equilibria of an arbitrary game tends to be enormous (see McLennan 2005), we emphasize the importance of computing a Nash equilibrium with a good game-theoretical underpinning. The algorithms of van den Elzen-Talman, Herings-van den Elzen, and Herings-Peeters are all related to the equilibrium selection 
methods of Harsanyi and Selten. The McKelvey-Palfrey algorithm has an interesting behavioral interpretation.

We have presented an overview of how homotopy methods have been applied to games in extensive form and to dynamic games, and how homotopy methods can be used to compute all Nash equilibria of a game. We think that these are still underexplored research areas and we expect more exciting work to be done in the future.

Open Access This article is distributed under the terms of the Creative Commons Attribution Noncommercial License which permits any noncommercial use, distribution, and reproduction in any medium, provided the original author(s) and source are credited.

\section{References}

Allgower, E.L., Georg, K.: Numerical Continuation Methods: An Introduction. Berlin: Springer (1990)

Avis, D., Rosenberg, G., Savani, R., von Stengel, B.: Enumeration of Nash equilibria for two-player games. Econ Theory (2009, this issue)

Balthasar, A.: Equilibrium tracing in strategic-form games. Econ Theory (2009, this issue)

Browder, F.E.: On continuity of fixed points under deformations of continuous mappings. Summa Brasiliensis Math 4, 183-191 (1960)

Datta, R.S.: Finding all Nash equilibria of a finite game using polynomial algebra. Econ Theory (2009, this issue)

Eaves, B.C.: Polymatrix games with joint constraints. SIAM J Appl Math 24, 418-423 (1973)

Eaves, B.C., Schmedders, K.: General equilibrium models and homotopy methods. J Econ Dyn Control 23, 1249-1279 (1999)

van den Elzen, A.H., Talman, A.J.J.: A procedure for finding Nash equilibria in bi-matrix games. ZOR Methods Models Oper Res 35, 27-43 (1991)

van den Elzen, A.H., Talman, A.J.J.: An algorithmic approach toward the tracing procedure for bi-matrix games. Games Econ Behav 28, 130-145 (1999)

Filar, J.A., Raghavan, T.E.S.: A matrix game solution of a single controller stochastic game. Math Oper Res 9, 356-362 (1984)

Garcia, C.B., Zangwill, W.I.: Pathways to Solutions, Fixed Points, and Equilibria. Englewood Cliffs: Prentice Hall (1981)

Garcia, C.B., Lemke, C.E., Lüthi, H.J.: Simplicial approximation of an equilibrium point of noncooperative $n$-person games. In: Hu, T.C., Robinson, S.M. (eds.) Mathematical Programming, pp. 227-260. New York: Academic Press (1973)

Geanakoplos, J.D.: Nash and Walras equilibrium via Brouwer. Econ Theory 21, 585-603 (2003)

Gilboa, I., Zemel, E.: Nash and correlated equilibria: Some complexity considerations. Games Econ Behav 1, 80-93 (1989)

Govindan, S., Wilson, R.: A global Newton method to compute Nash equilibria. J Econ Theory 110, 65-86 (2003)

Govindan, S., Wilson, R.: Computing Nash equilibria by iterated polymatrix approximation. J Econ Dyn Control 28, 1229-1241 (2004)

Harsanyi, J.C.: The tracing procedure: a Bayesian approach to defining a solution for $n$-person noncooperative games. Int J Game Theory 4, 61-94 (1975)

Harsanyi, J.C., Selten, R.: A General Theory of Equilibrium Selection in Games. Cambridge: MIT Press (1988)

Herings, P.J.J.: A globally and universally stable price adjustment process. J Math Econ 27, 163-193 (1997)

Herings, P.J.J.: Two simple proofs of the feasibility of the linear tracing procedure. Econ Theory 15, 485$490(2000)$

Herings, P.J.J.: Universally converging adjustment processes—a unifying approach. J Math Econ 38, 341370 (2002)

Herings, P.J.J., van den Elzen, A.H.: Computation of the Nash equilibrium selected by the tracing procedure in $n$-person games. Games Econ Behav 38, 89-117 (2002)

Herings, P.J.J., Peeters, R.: A differentiable homotopy to compute Nash equilibria of $n$-person games. Econ Theory 18, 159-185 (2001) 
Herings, P.J.J., Peeters, R.: Stationary equilibria in stochastic games: structure, selection and computation. J Econ Theory 118, 32-60 (2004)

Herings, P.J.J., Peeters, R.: A globally convergent algorithm to compute all Nash equilibria for $n$-person games. Ann Oper Res 137, 349-368 (2005)

Howson, J.T. Jr.: Equilibria of polymatrix games. Manag Sci 21, 313-315 (1972)

Howson, J.T. Jr., Rosenthal, R.W.: Bayesian equilibria of finite two-person games with incomplete information. Manag Sci 21, 313-315 (1974)

Jongen, H.Th., Jonker, P., Twilt, F.: Nonlinear optimization in $\mathbb{R}^{n}$, I. Morse Theory, Chebyshev Approximation. Methoden und Verfahren der Mathematischen Physik, 29. Frankfurt: Peter Lang (1983)

Judd, K.L.: Computational economics and economic theory: substitutes or complements? J Econ Dyn Control 21, 907-942 (1997)

Kohlberg, E., Mertens, J.F.: On the strategic stability of equilibria. Econometrica 54, 1003-1037 (1986)

Koller, D., Megiddo, N., von Stengel, B.: Efficient computation of equilibria for extensive two-person games. Games Econ Behav 14, 247-259 (1996)

Kostreva, M.M., Kinard, L.A.: A differential homotopy approach for solving polynomial optimization problems and noncooperative games. Comput Math Appl 21, 135-143 (1991)

van der Laan, G., Talman, A.J.J., van der Heijden, L.: Simplicial variable dimension algorithms for solving the nonlinear complementarity problem on a product of unit simplices using a general labelling. Math Oper Res 12, 377-397 (1987)

Lemke, C.E.: Bimatrix equilibrium points and mathematical programming. Manag Sci 11, 681-689 (1965)

Lemke, C.E., Howson, J.T. Jr.: Equilibrium points of bimatrix games. SIAM J Appl Math 12, 413-423 (1964)

Mas-Colell, A.: A note on a theorem of F. Browder. Math Program 6, 229-233 (1974)

Mas-Colell, A.: The Theory of General Economic Equilibrium, a Differentiable Approach. Cambridge: Cambridge University Press (1985)

McKelvey, R.D., McLennan, A.: Computation of equilibria in finite games. In: Amman, H.M., Kendrick, D.A., Rust, J. (eds.) Handbook of Computational Economics, vol. I, pp. 87-142. Amsterdam: Elsevier/North-Holland (1996)

McKelvey, R.D., Palfrey, T.R.: Quantal response equilibria for normal form games. Games Econ Behav 10, 6-38 (1995)

McKelvey, R.D., Palfrey, T.R.: Quantal response equilibria for extensive form games. Exp Econ 1, 9-41 (1998)

McLennan, A.: The expected number of Nash equilibria of a normal form game. Econometrica 73, 141-174 (2005)

Nowak, A.S., Raghavan, T.E.S.: A finite step algorithm via a bimatrix game to a single controller nonzerosum stochastic game. Math Program 59, 249-259 (1993)

Raghavan, T.E.S., Syed, Z.: Computing stationary Nsh equilibria of undiscounted single-controller stochastic games. Math Oper Res 22, 384-400 (2002)

Rosenmüller, J.: On a generalization of the Lemke-Howson algorithm to noncooperative $n$-person games. SIAM J Appl Math 21, 73-79 (1971)

Rosenthal, R.W.: A bounded-rationality approach to the study of noncooperative games. Int J Game Theory 18, 273-292 (1989)

Schanuel, S.H., Simon, L.K., Zame, W.R.: The algebraic geometry of games and the tracing procedure. In: Selten, R. (ed.) Game Equilibrium Models II: Methods, Morals and Markets, pp. 9-43. Berlin: Springer (1991)

Shapley, L.S.: A note on the Lemke-Howson algorithm. Mathematical programming study. Pivoting Ext 1, 175-189 (1974)

von Stengel, B.: Efficient computation of behavior strategies. Games Econ Behav 14, 220-246 (1996)

von Stengel, B.: Computing equilibria for two-person games. In: Aumann, R.J., Hart, S. (eds.) Handbook of Game Theory, chap. 45, vol. 3, pp. 1723-1759. Amsterdam: North-Holland (2002)

von Stengel, B., van den Elzen, A.H., Talman, A.J.J.: Computing normal form perfect equilibria for extensive two-person games. Econometrica 70, 693-715 (2002)

Sturmfels, B.: Solving Systems of Polynomial Equations. American Mathematical Society, CBMS Regional Conferences Series, No. 97. Rhode Island, Providence (2002)

Todd, M.J.: The computation of fixed points and applications. Lecture Notes in Economics and Mathematical Systems, vol. 124. Berlin: Springer (1976)

Turocy, T.L.: A dynamic homotopy interpretation of the logistical quantal response equilibrium correspondence. Games Econ Behav 51, 243-263 (2005) 
Voorneveld, M.: Probabilistic choice in games: properties of Rosenthal's $t$-solutions. Int J Game Theory 34, 105-122 (2006)

Watson, L.T., Billups, S.C., Morgan, A.P.: HOMPACK: a suite of codes for globally convergent homotopy algorithms. ACM Trans Math Softw 13, 281-310 (1987)

Wilson, R.: Computing equilibria of $n$-person games. SIAM J Appl Math 21, 80-87 (1971)

Wilson, R.: Computing equilibria of two-person games from the extensive form. Manag Sci 18, 448-460 (1972)

Wilson, R.: Computing simply stable equilibria. Econometrica 60, 1039-1070 (1992)

Yamamoto, Y.: A path-following procedure to find a proper equilibrium of finite games. Int J Game Theory 22, 249-259 (1993)

Yanovskaya, E.B.: Equilibrium points in polymatrix games. Litovskii Matematicheskii Sbornik 8, 381-384 (1986) (in Russian) (see also Math Rev 39, \# 3831) 